Claire Voisin

\title{
Green's generic syzygy conjecture for curves of even genus lying on a $K 3$ surface
}

Received June 6, 2001 / final version received January 30, 2002

Published online July 9, 2002 - (C) Springer-Verlag \& EMS 2002

\section{Introduction}

If $C$ is a smooth projective curve of genus $g$ and $K_{C}$ is its canonical bundle, the theorem of Noether asserts that the multiplication map

$$
\mu_{0}: H^{0}\left(C, K_{C}\right) \otimes H^{0}\left(C, K_{C}\right) \rightarrow H^{0}\left(C, K_{C}^{\otimes 2}\right)
$$

is surjective when $C$ is non hyperelliptic.

The theorem of Petri concerns then the ideal $I$ of $C$ in its canonical embedding, assuming $C$ is not hyperelliptic. It says that $I$ is generated by its elements of degree 2 if $C$ is neither trigonal nor a plane quintic.

In [7], M. Green introduced and studied the Koszul complexes

$$
\begin{aligned}
\bigwedge^{p+1} H^{0}(X, L) \otimes H^{0}\left(X, L^{q-1}\right) & \stackrel{\delta}{\rightarrow} \bigwedge^{p} H^{0}(X, L) \otimes H^{0}\left(X, L^{q}\right) \\
& \stackrel{\delta}{\rightarrow} \bigwedge_{p-1} H^{0}(X, L) \otimes H^{0}\left(X, L^{q+1}\right)
\end{aligned}
$$

for $X$ a variety and $L$ a line bundle on $X$. Denoting by $K_{p, q}(X, L)$ the cohomology at the middle of the sequence above, one sees immediately that the surjectivity of the map $\mu_{0}$ is equivalent to $K_{0,2}\left(C, K_{C}\right)=0$, and that if this is the case, the ideal $I$ is generated by quadrics if and only if $K_{1,2}\left(C, K_{C}\right)=0$. On the other hand, $C$ being non hyperelliptic is equivalent to the fact that the Clifford index Cliff $C$ is strictly positive, where

Cliff $C:=\operatorname{Min}\left\{d-2 r, \exists L \in \operatorname{Pic} C, d^{0} L=d, h^{0}(L)=r+1 \geq 2, h^{1}(L) \geq 2\right\}$.

Similarly, $C$ is neither hyperelliptic, nor trigonal nor a plane quintic if and only if Cliff $C>1$.

Green's conjecture on syzygies of canonical curves generalizes then the theorems of Noether and Petri as follows

Conjecture 1. [7] For a smooth projective curve $C$ in characteristic 0 , the condition Cliff $C>l$ is equivalent to the fact that $K_{l^{\prime}, 2}\left(C, K_{C}\right)=0, \forall l^{\prime} \leq l$.

C. Voisin: Institut de mathématiques de Jussieu, CNRS, UMR 7586, 175 rue du Chevaleret, 75013 Paris, France; e-mail: voisin@ math.jussieu.fr

Mathematics Subject Classification (2000): 14H51, 14J28, 14N99 
The interest of this formulation of Noether and Petri's theorems is already illustrated in [9], where these theorems are given a modern proof, using geometric technics of computation of syzygies.

For our purpose, and as is done in [7], it is convenient to use the duality (cf [7])

$$
K_{p, 2}\left(C, K_{C}\right) \cong K_{g-p-2,1}\left(C, K_{C}\right)^{*}
$$

to reformulate the conjecture as follows

Conjecture 2. [7] For a smooth projective curve $C$ of genus $g$ in characteristic 0 , the condition Cliff $C>l$ is equivalent to the fact that $K_{g-l^{\prime}-2,1}\left(C, K_{C}\right)=0$, $\forall l^{\prime} \leq l$.

If $C$ is now a generic curve, the theorem of Brill-Noether (cf [2], [11]) implies that

$$
\text { Cliff } C=\operatorname{gon}(\mathrm{C})-2
$$

where the gonality $\operatorname{gon}(\mathrm{C}):=\operatorname{Min}\left\{d, \exists L \in \operatorname{Pic} C, d^{0} L=d, h^{0}(L) \geq 2\right\}$, and that

$$
\begin{aligned}
& \operatorname{gon}(\mathrm{C})=\frac{g+3}{2}, \text { if } g \text { is odd, } \\
& \operatorname{gon}(\mathrm{C})=\frac{g+2}{2}, \text { if } g \text { is even. }
\end{aligned}
$$

Hence we arrive at the following conjecture (the generic Green conjecture on syzygies of a canonical curve):

Conjecture 3. Let $C$ be a generic curve of genus $g$. Then if $g=2 k+1$ or $g=2 k$, we have $K_{k, 1}\left(C, K_{C}\right)=0$.

Remark 1. The actual conjecture is $K_{l, 1}\left(C, K_{C}\right)=0, \forall l \geq k$; but it is easy to prove that

$$
K_{k, 1}\left(C, K_{C}\right)=0 \Rightarrow K_{l, 1}\left(C, K_{C}\right)=0, \forall l \geq k .
$$

Notice that in the appendix to [7], Green and Lazarsfeld prove the Conjecture 1 in the direction $\Leftarrow$ (i. e. they produce non zero syzygies from special linear systems.) Hence the conjecture above cannot be improved, namely, under the assumptions above, we have $K_{k-1,1}\left(C, K_{C}\right) \neq 0$.

Teixidor [16] has recently proposed an approach to the Conjecture 3. Her method uses a degeneration to a tree of elliptic curves and the theory of limit linear series of Eisenbud and Harris [6], adapted to vector bundles of higher rank. It is very likely that her method will lead to a proof of the generic Green conjecture.

We propose here a completely different approach, which does not prove Conjecture 3 in odd genus, but proves Green's conjecture 2 for generic curves $C$ of genus $g(C)$ and gonality gon $(\mathrm{C})$ in the range

$$
\frac{g(C)}{3}+1 \leq \operatorname{gon}(\mathrm{C}) \leq \frac{g(C)}{2}+1 .
$$

The inequality on the left says that the gonality has to be not too small, but the only case which is excluded by the inequality on the right is that of a generic curve of 
odd genus $g$, which has gonality $\frac{g+3}{2}$. Combined with the results of Teixidor [17], this shows that Green's conjecture is true for any generic curve of given gonality, except for the generic curve of odd genus.

Recall from [11] that if $S$ is a $K 3$ surface endowed with a ample line bundle $L$ such that $L$ generates Pic $S$ and $L^{2}=2 g-2$, the smooth members $C \in|L|$ are of genus $g$ and generic in the sense of Brill-Noether, so that in particular they have the same Clifford index as a generic curve. Hence Conjecture 1 predicts that their syzygies vanish as stated in Conjecture 3. This is indeed what we prove here, in the case where the genus is even. Note first that the hyperplane restriction theorem [7] says that

$$
K_{k, 1}\left(C, K_{C}\right)=K_{k, 1}(S, L)
$$

whenever $C$ is a hyperplane section of a $K 3$ surface $S$ (note that $K_{C}=L_{\mid C}$ in this case). Conjecture 3 for curves of even genus is therefore implied by

Theorem 1. The pair $(S, L)$ being as above, with $g=2 k$, we have

$$
K_{k, 1}(S, L)=0 .
$$

The body of the paper will be devoted to the proof of (1.2). It turns out that Theorem 1 in turn has much stronger consequences than the generic syzygy conjecture for curves of even genus, and we shall explain this now. In fact we have the following corollary:

Corollary 1. For any $\delta \leq \frac{k}{2}$, the generic curve of genus $2 k-\delta$ which is $k+1-\delta$ gonal satisfies

$$
K_{k, 1}\left(C, K_{C}\right)=0
$$

or equivalently by the duality theorem

$$
K_{k-\delta-2,2}\left(C, K_{C}\right)=0 .
$$

Notice that this result is optimal and exactly predicted by Green's Conjecture 1, since the Clifford index of such curve is less than or equal to $k-1-\delta$. An easy computation shows that the pairs

$$
g=2 k-\delta, \text { gon }=\mathrm{k}+1-\delta, \mathrm{k} \geq 0, \delta \leq \frac{\mathrm{k}}{2}
$$

are exactly the pairs satisfying the inequalities

$$
\frac{g}{3}+1 \leq \text { gon } \leq \frac{g}{2}+1
$$

Hence Green's conjecture is proved for generic curves of genus and gonality in this range. 
Proof of Corollary 1. Let $(S, L)$ be as in Theorem 1. A generic member $X$ of $|L|$ is $k+1$-gonal. As in Sect. 2, and following [11], it follows that there is a rank 2 vector bundle $E$ on $S$ with det $E=L, c_{2}(E)=k+1$, and $h^{0}(E)=k+2$. The zero set of a generic section of $E$ is a generic member of a $g_{k+1}^{1}$ of a generic curve $X \in|L|$.

Now let $x_{1}, \ldots, x_{\delta}$ be generic points of $S$. Beacause $\delta \leq \frac{k}{2}$, the space

$$
H_{x .}=H^{0}\left(S, E \otimes \mathcal{I}_{x_{1}} \otimes \ldots \otimes \mathcal{I}_{x_{\delta}}\right)
$$

has rank at least 2. One checks that for generic $x_{i}$ 's, and for $\alpha, \beta$ generic in this space, the curve $X$ defined by the equation

$$
\operatorname{det}(\alpha \wedge \beta) \in H^{0}(S, \operatorname{det} E)=H^{0}(S, L)
$$

is nodal with nodes exactly as the $x_{i}$ 's. On the other hand, the two sections $\alpha, \beta$ generate a rank 1 subsheaf of the restriction $E_{\mid X}$. Let now

$$
n: C \rightarrow X
$$

be the normalization. The rank 1 subsheaf introduced above induces a line subbundle

$$
D \subset n^{*} E
$$

with two sections, and it is obvious that the moving part of this linear system on $C$ is of degree $k+1-\delta$, since the sections $\lambda \alpha+\mu \beta$ of $E$ vanish at the $x_{i}$ 's, so that the moving part of their zero sets is of degree $k+1-\delta$. Hence $C$ is $k+1-\delta$-gonal. It remains to show that

$$
K_{k, 1}\left(C, K_{C}\right)=0
$$

Now we have by the hyperplane restriction theorem and by Theorem 1 the vanishing

$$
K_{k, 1}\left(X, K_{X}\right)=0 \text {. }
$$

We prove now that this implies (1.3). Notice that there is an identification of $H^{0}\left(C, K_{C}\right)$ with a subspace of $H^{0}\left(X, K_{X}\right)$, namely the last space is a space of meromorphic forms on $C$ with logarithmic singularities over the nodes satisfying the condition that the sum of the residues over each node vanishes. $H^{0}\left(C, K_{C}\right)$ is then the subspace of forms which are regular.

From this inclusion $j: H^{0}\left(C, K_{C}\right) \hookrightarrow H^{0}\left(X, K_{X}\right)$, we can deduce a commutative diagram of Koszul complexes

$$
\begin{aligned}
\bigwedge^{k+1} H^{0}\left(C, K_{C}\right) & \rightarrow \bigwedge^{k} H^{0}\left(C, K_{C}\right) \otimes H^{0}\left(C, K_{C}\right) \\
j \downarrow & \rightarrow \bigwedge^{k-1} H^{0}\left(C, K_{C}\right) \otimes H^{0}\left(C, K_{C}^{\otimes 2}\right) \\
j \downarrow & j \downarrow \\
\bigwedge^{k+1} H^{0}\left(X, K_{X}\right) & \rightarrow \bigwedge^{k} H^{0}\left(X, K_{X}\right) \otimes H^{0}\left(X, K_{X}\right) \rightarrow \bigwedge^{k-1} H^{0}\left(X, K_{X}\right) \otimes H^{0}\left(X, K_{X}^{\otimes 2}\right) .
\end{aligned}
$$


We claim that this induces an inclusion

$$
j: K_{k, 1}\left(C, K_{C}\right) \hookrightarrow K_{k, 1}\left(X, K_{X}\right) .
$$

Indeed, consider in general the Koszul differential

$$
\delta: \bigwedge^{l} H^{0}(Y, \mathcal{L}) \rightarrow H^{0}(Y, \mathcal{L}) \otimes \bigwedge^{l-1} H^{0}(Y, \mathcal{L})
$$

Then if

$$
\wedge: H^{0}(Y, \mathcal{L}) \otimes \bigwedge^{l-1} H^{0}(Y, \mathcal{L}) \rightarrow \bigwedge^{l} H^{0}(Y, \mathcal{L})
$$

is the wedge product map, one has

$$
\wedge \circ \delta= \pm l I d
$$

Consider now the inclusion

$$
j: H^{0}\left(C, K_{C}\right) \otimes \bigwedge^{k} H^{0}\left(C, K_{C}\right) \rightarrow H^{0}\left(X, K_{X}\right) \otimes \bigwedge^{k} H^{0}\left(X, K_{X}\right) .
$$

Let $\alpha \in H^{0}\left(C, K_{C}\right) \otimes \bigwedge^{k} H^{0}\left(C, K_{C}\right)$ such that $\delta \alpha=0$ and $j(\alpha)=\delta \beta$. Then (1.5) gives

$$
\begin{aligned}
j(\alpha) & =\delta \beta= \pm \frac{1}{k+1} \delta(\wedge \circ \delta \beta) \\
& = \pm \frac{1}{k+1} \delta(\wedge(j(\alpha))) .
\end{aligned}
$$

But $\wedge(j(\alpha))=j(\wedge \alpha)$, so that this implies by injectivity of $j$ that $\alpha= \pm \frac{1}{k+1} \delta(\wedge \alpha)$. Hence $\alpha$ is in fact exact. Hence our claim is proven.

In the missed case of a generic curve of odd genus, we have the following corollary:

Corollary 2. Let $C$ be a generic curve of genus $g=2 k-1$; then

$$
K_{k, 1}\left(C, K_{C}\right)=0 .
$$

(Notice that the generic Green conjecture predicts in fact that $K_{k-1,1}\left(C, K_{C}\right)=0$.)

Proof of Corollary 2. The $K 3$ surface $S$ being as above, let $X$ be a member of $|L|$ with exactly one node as singularity. Let $C$ be the normalization of $X$. Then the genus of $C$ is equal to $2 k-1$.

We have as before an inclusion

$$
H^{0}\left(C, K_{C}\right) \hookrightarrow H^{0}\left(X, K_{X}\right)
$$

which induces by the same argument as in the proof of Corollary 1 an inclusion

$$
K_{k, 1}\left(C, K_{C}\right) \hookrightarrow K_{k, 1}\left(X, K_{X}\right) .
$$


The hyperplane restriction theorem can be applied to $X \subset S$, and together with the vanishing (1.2), it gives

$$
K_{k, 1}\left(X, K_{X}\right)=0
$$

Hence $K_{k, 1}\left(C, K_{C}\right)=0$.

We conclude this introduction with a sketch of the main ideas in the proof of Theorem 1 . The very starting point is the following observation: denote by $S^{[l]}$ the Hilbert scheme parametrizing 0-dimensional length $l$ subschemes of $S$. Let $I_{l} \subset S \times S^{[l]}$ be the incidence subscheme and

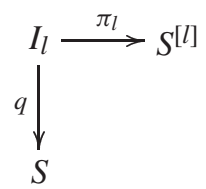

be the incidence correspondence. Let

$$
\mathcal{E}_{L}:=R^{0} \pi_{l *} q^{*} L
$$

and $L_{l}:=\operatorname{det} \mathcal{E}_{L}$. Then we have

Fact. $K_{l-1,1}(S, L)=0$ if and only if

$$
H^{0}\left(I_{l}, \pi_{l}^{*} L_{l}\right)=\pi_{l}^{*} H^{0}\left(S^{[l]}, L_{l}\right) .
$$

Our strategy will be then to construct a subvariety $Z$ of $S^{[k+1]}$, such that

$$
H^{0}\left(\tilde{Z}, \pi_{l}^{*} L_{l}\right)=\pi_{l}^{*} H^{0}\left(Z, L_{l}\right)
$$

where $\tilde{Z}:=\pi_{l}^{-1}(Z)$, and the restriction map

$$
H^{0}\left(I_{l}, \pi_{l}^{*} L_{l}\right) \rightarrow H^{0}\left(\tilde{Z}, \pi_{l}^{*} L_{l}\right)
$$

is injective.

As in the papers [11], [8], the key role in constructing our variety $Z$ and verifying the conditions above will be played by the Lazarsfeld-Mukai vector bundle on $S$ associated with minimal degree base-point free linear systems on smooth members of $|L|$.

Terminology. In this paper, we shall say that a Zariski open subset $U \subset X$ is large if the complementary closed subset $Z=X-U$ has codimension non smaller that 2 in $X$. In the considered cases, the variety $X$ will be normal, and we will use freely the fact that for a line bundle $\mathcal{L}$ on $X$

$$
H^{0}(X, \mathcal{L}) \cong H^{0}\left(U, \mathcal{L}_{\mid U}\right)
$$

for $U$ a large open subset of $X$. 


\section{Strategy of the proof}

We start with the following observation: Let $X$ be a smooth projective variety. Denote by $X_{\text {curv }}^{[k]}$ the Hilbert scheme parametrizing curvilinear 0-dimensional subschemes of $X$ of length $k . X_{\text {curv }}^{[k]}$ is smooth, and if $X$ is a curve or a surface, it is a large open set in the Hilbert scheme $X^{[k]}$ which is smooth.

Let

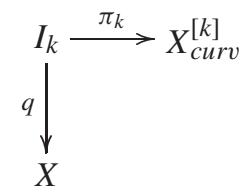

be the incidence correspondence. For a line bundle $L$ on $X$ denote by $\mathcal{E}_{L}$ the vector bundle on $X_{\text {curv }}^{[k]}$ defined by $\mathcal{E}_{L}=R^{0} \pi_{k *} q^{*} L$, and let

$$
L_{k}:=\operatorname{det} \mathcal{E}_{L} .
$$

We have

Lemma 1. There is a natural isomorphism

$$
K_{k, 1}(X, L) \cong H^{0}\left(I_{k+1}, \pi_{k+1}^{*} L_{k+1}\right) / \pi_{k+1}^{*} H^{0}\left(X_{\text {curv }}^{[k+1]}, L_{k+1}\right) .
$$

In particular, $K_{k, 1}(X, L)=0$ is equivalent to

$$
H^{0}\left(I_{k+1}, \pi_{k+1}^{*} L_{k+1}\right)=\pi_{k+1}^{*} H^{0}\left(X_{c u r v}^{[k+1]}, L_{k+1}\right) .
$$

Proof. Recall that $K_{k, 1}(X, L)$ is the cohomology at the middle of the sequence

$$
\bigwedge_{k+1} H^{0}(X, L) \stackrel{\delta}{\rightarrow} H^{0}(X, L) \otimes \bigwedge^{k} H^{0}(X, L) \stackrel{\delta}{\rightarrow} H^{0}\left(X, L^{\otimes 2}\right) \otimes \bigwedge^{k-1} H^{0}(X, L) .
$$

Now note that there is a natural morphism

$$
\tau: I_{k+1} \rightarrow X \times X_{c u r v}^{[k]}
$$

which to $(x, z), x \in \operatorname{Supp} z$ associates $\left(x, z^{\prime}\right)$, where $z^{\prime}$ is the residual scheme of $x$ in $z$. This morphism is well defined because we are working with curvilinear schemes.

One shows easily that $\tau$ identifies $I_{k+1}$ to a large open subset of the blow-up of $X \times X_{\text {curv }}^{[k]}$ along the incidence subscheme $I_{k}$. (Indeed, away from $I_{k}$ the inverse $\tau^{-1}$ of $\tau$ is given by

$$
\left.\tau^{-1}((x, z))=x \cup z, x \in X, z \in X_{c u r v}^{[k]}\right)
$$

Furthermore, if $D \subset I_{k+1}$ is the exceptional divisor one has

$$
\pi_{k+1}^{*} L_{k+1}=\tau^{*}\left(L \otimes L_{k}\right)(-D) .
$$


This isomorphism is obtained by studying the natural morphism of vector bundles over $I_{k+1}$

$$
\pi_{k+1}^{*} \mathcal{E}_{L} \rightarrow \tau^{*}\left(p r_{1}^{*} L \oplus p r_{2}^{*} \mathcal{E}_{L}\right),
$$

which at a point $\tilde{z}$ such that $\tau(\tilde{z})=\left(x, z^{\prime}\right)$ identifies to the restriction map

$$
H^{0}\left(L_{\mid \tilde{z}}\right) \rightarrow L_{\mid x} \oplus H^{0}\left(L_{\mid z^{\prime}}\right) .
$$

It is immediate to see that the cokernel of this morphism is supported on the exceptional divisor $D$ of $\tau$ and is of rank 1 on $D$.

It follows that

$$
\begin{aligned}
& H^{0}\left(I_{k+1}, \pi_{k+1}^{*} L_{k+1}\right) \\
& \quad=\operatorname{Ker}\left(H^{0}(X, L) \otimes H^{0}\left(X_{\text {curv }}^{[k]}, L_{k}\right) \stackrel{\text { rest }}{\rightarrow} H^{0}\left(I_{k}, L \otimes L_{k \mid I_{k}}\right)\right) .
\end{aligned}
$$

We now apply the description above to $I_{k}$ : we note that denoting by $p_{i}, i=$ 1,2 , the compositions of the projections with the inclusion $I_{k} \hookrightarrow X \times X_{c u r v}^{[k]}$, we have

$$
p_{2}=\pi_{k}, p_{1}=p r_{1} \circ \tau^{\prime}
$$

where

$$
\tau^{\prime}: I_{k} \rightarrow X \times X_{c u r v}^{[k-1]}
$$

is defined as in (2.8). Hence applying formula (2.9), we get

$$
L \otimes L_{k \mid I_{k}}=\tau^{*}\left(L^{2} \otimes L_{k-1}\right)\left(-D^{\prime}\right),
$$

where $D^{\prime}$ is now the exceptional divisor of the blowing-down morphism $\tau^{\prime}: I_{k} \rightarrow$ $X \times X_{\text {curv }}^{[k-1]}$. So we conclude that there is a natural inclusion

$$
i: H^{0}\left(I_{k}, L \otimes L_{k \mid I_{k}}\right) \subset H^{0}\left(X, L^{\otimes 2}\right) \otimes H^{0}\left(X_{\text {curv }}^{[k-1]}, L_{k-1}\right) .
$$

Hence we have constructed from formula (2.10) an exact sequence

$$
\begin{aligned}
& 0 \rightarrow H^{0}\left(I_{k+1}, \pi_{k+1}^{*} L_{k+1}\right) \stackrel{j}{\rightarrow} H^{0}(X, L) \otimes H^{0}\left(X_{\text {curv }}^{[k]}, L_{k}\right) \\
& \stackrel{i \circ \text { rest }}{\rightarrow} H^{0}\left(X, L^{\otimes 2}\right) \otimes H^{0}\left(X_{\text {curv }}^{[k-1]}, L_{k-1}\right) .
\end{aligned}
$$

Next, it is a standard fact that the natural map

$$
\bigwedge^{l} H^{0}(X, L) \rightarrow H^{0}\left(X_{c u r v}^{[l]}, L_{l}\right)
$$

induced by the evaluation map

$$
H^{0}(X, L) \otimes \mathcal{O}_{X_{\text {curv }}^{[k]}} \rightarrow \mathcal{E}_{L}
$$

are isomorphisms for any $l$. To check this, one considers the large open subset $U^{[l]}$ of $X_{\text {curv }}^{[l]}$, made of subschemes $z$ which have at most one point of multiplicity 2 as singularity. This set $U^{[l]}$ has the following description: one considers inside $X^{l}$ the large open set $X_{0}^{l}$ made of $l$-uples $\left(x_{1}, \ldots, x_{l}\right)$, where at most two of the $x_{i}$ 's 
coincide. Inside $X_{0}^{l}$, one blows-up the generalized diagonal $\cup_{i \neq j}\left\{x_{i}=x_{j}\right\}$, which is smooth there, and one takes the quotient of the resulting variety $\widetilde{X_{0}^{l}}$ by the action of the symmetric group $S_{l}$. By the same argument as above one finds that if

$$
r: \widetilde{X_{0}^{l}} \rightarrow U^{[l]}
$$

is the quotient map, one has

$$
r^{*} L_{l} \cong L \otimes \ldots \otimes L(-E),
$$

where $E$ is the exceptional divisor of the blowing-up map $\tau^{\prime \prime}: \widetilde{X_{0}^{l}} \rightarrow X_{0}^{l}$. It follows that one has an identification

$$
H^{0}\left(X_{\text {curv }}^{[l]}, L_{l}\right)=H^{0}\left(U^{[l]}, L_{l}\right) \cong H^{0}\left(\tilde{X}_{0}^{l}, \tau^{\prime \prime *}\left(\otimes_{i=1}^{i=l} L\right)(-E)\right)^{S_{l}} .
$$

The last space is a subspace of

$$
H^{0}\left(X_{0}^{l}, \bigotimes_{i=1}^{i=l} L\right)^{S_{l}} \cong H^{0}\left(X^{l}, \bigotimes_{i=1}^{i=l} L\right)^{S_{l}}=\left(\otimes_{i=1}^{i=l} H^{0}(X, L)\right)^{S_{l}} .
$$

But looking more closely at the action of $S_{l}$ on the line bundle $r^{*} L_{k}$, one verifies that the induced action of $S_{l}$ on $\otimes_{i=1}^{i=l} H^{0}(X, L)$ is the twisted action. Hence the invariant subspace

$$
\left(\otimes_{i=1}^{i=l} H^{0}(X, L)\right)^{S_{l}}
$$

is isomorphic to $\bigwedge^{l} H^{0}(X, L)$. One verifies then that the injective map

$$
H^{0}\left(X_{\text {curv }}^{[l]}, L_{l}\right) \rightarrow \bigwedge^{l} H^{0}(X, L)
$$

so obtained is a left inverse for the map (2.11).

Using the isomorphisms (2.11), the exact sequence above becomes

$$
\begin{aligned}
& 0 \rightarrow H^{0}\left(I_{k+1}, \pi_{k+1}^{*} L_{k+1}\right) \stackrel{j}{\rightarrow} H^{0}(X, L) \otimes \bigwedge^{k} H^{0}(X, L) \\
& \stackrel{i \circ \text { rest }}{\rightarrow} H^{0}\left(X, L^{\otimes 2}\right) \otimes \bigwedge^{k-1} H^{0}(X, L),
\end{aligned}
$$

hence it remains only to show that the maps $j \circ \pi_{k+1}^{*}$ and $i \circ$ rest identify via the isomorphisms (2.11) to the differentials $\delta$ of the sequence (2.7), which is easy.

We consider now a $K 3$ surface $S$ endowed with an ample line bundle $L$ generating Pic $S$ and satisfying

$$
L^{2}=2 g-2, g=2 k, k>1 .
$$

We now explain our strategy to prove the vanishing

$$
K_{k, 1}(S, L)=0 .
$$

Assume we have a subscheme $T \subset S^{[k+1]}$ such that, if $\tilde{T}$ denotes the subvariety $\pi_{k+1}^{-1}(T)$ of $I_{k+1}$, the following conditions are satisfied: 
1. We have an isomorphism

$$
H^{0}\left(\tilde{T}, \pi_{k+1}^{*} L_{k+1}\right)=\pi_{k+1}^{*} H^{0}\left(T, L_{k+1}\right) .
$$

2. The restriction map

$$
H^{0}\left(I_{k+1}, \pi_{k+1}^{*} L_{k+1}\right) \rightarrow H^{0}\left(\tilde{T}, \pi_{k+1}^{*} L_{k+1}\right)
$$

is injective.

Then we claim that $K_{k, 1}(S, L)=0$.

Indeed we have the trace maps

$$
\begin{gathered}
t r: H^{0}\left(I_{k+1}, \pi_{k+1}^{*} L_{k+1}\right) \rightarrow H^{0}\left(S_{c u r v}^{[k+1]}, L_{k+1}\right) \\
\operatorname{tr}_{T}: H^{0}\left(\tilde{T}, \pi_{k+1}^{*} L_{k+1}\right) \rightarrow H^{0}\left(T, L_{k+1}\right)
\end{gathered}
$$

which commute with the restriction maps and which compose to $(k+1) I d$ with the pull-back maps. If $\sigma \in H^{0}\left(I_{k+1}, \pi^{*} L_{k+1}\right)$, there exists by property 1 an element $\beta \in H^{0}\left(T, L_{k+1}\right)$ such that

$$
\sigma_{\mid \tilde{T}}=\pi_{k+1}^{*} \beta .
$$

Then

$$
\beta=\frac{1}{k+1} \operatorname{tr}_{T}\left(\sigma_{\mid \tilde{T}}\right)=\left(\frac{1}{k+1} \operatorname{tr} \sigma\right)_{\mid T} .
$$

Hence the section

$$
\sigma^{\prime}=\sigma-\pi_{k+1}^{*}\left(\frac{1}{k+1} \operatorname{Tr} \sigma\right)
$$

vanishes on $\tilde{T}$, hence it is zero by property 2 . So we have

$$
H^{0}\left(I_{k+1}, \pi_{k+1}^{*} L_{k+1}\right)=\pi_{k+1}^{*} H^{0}\left(S_{\text {curv }}^{[k+1]}, L_{k+1}\right)
$$

and this proves our claim, using Lemma 1.

We will have to weaken the assumptions above as follows: Suppose we have a normal scheme $Z$ together with a morphism

$$
j: Z \rightarrow I_{k+1}
$$

such that $\pi_{k+1} \circ j$ is generically one to one on its image, which is not contained in the branch locus of $\pi_{k+1}$. Suppose also that we have a normal scheme $Z^{\prime}$ together with a proper degree $k$ morphism $\pi^{\prime}: Z^{\prime} \rightarrow Z$ and a morphism $j^{\prime}: Z^{\prime} \rightarrow I_{k+1}$ satisfying the conditions that

$$
\pi_{k+1} \circ j^{\prime}=j \circ \pi^{\prime}
$$

and the union $j(Z) \cup j^{\prime}\left(Z^{\prime}\right)$ is equal set theoretically to $\pi_{k+1}^{-1}\left(\pi_{k+1} \circ j(Z)\right)$. Finally assume there are subschemes $Z_{1}^{\prime} \subset Z^{\prime}, Z_{1} \subset Z$ such that

$$
\pi_{\mid Z_{1}^{\prime}}^{\prime}=: \phi: Z_{1}^{\prime} \rightarrow Z_{1}
$$

is a birational isomorphism and $j \circ \phi=j_{\mid Z_{1}^{\prime}}^{\prime}$.

(Hence roughly speaking, and up to birational maps, $\pi_{k+1}^{-1}\left(\pi_{k+1} \circ j(Z)\right)$ is the scheme obtained by gluing $Z^{\prime}$ and $Z$ along $Z_{1}^{\prime} \cong Z_{1}$.) 
Assume now that they satisfy the following set $(\mathrm{H})$ of hypotheses

1. The map

$$
\pi^{\prime *}: H^{0}\left(Z,\left(\pi_{k+1} \circ j\right)^{*} L_{k+1}\right) \rightarrow H^{0}\left(Z^{\prime},\left(\pi_{k+1} \circ j^{\prime}\right)^{*} L_{k+1}\right)
$$

is an isomorphism.

2. The restriction map

$$
H^{0}\left(Z,\left(\pi_{k+1} \circ j\right)^{*} L_{k+1}\right) \rightarrow H^{0}\left(Z_{1},\left(\pi_{k+1} \circ j\right)^{*} L_{k+1 \mid Z_{1}}\right)
$$

is injective.

3. The restriction map

$$
j^{*}: H^{0}\left(I_{k+1}, \pi_{k+1}^{*} L_{k+1}\right) \rightarrow H^{0}\left(Z,\left(\pi_{k+1} \circ j\right)^{*} L_{k+1}\right)
$$

is injective.

Then we claim that $K_{k, 1}(S, L)=0$.

Indeed by Lemma 1 we have to show that

$$
H^{0}\left(I_{k+1}, \pi_{k+1}^{*} L_{k+1}\right)=\pi_{k+1}^{*} H^{0}\left(S_{\text {curv }}^{[k+1]}, L_{k+1}\right) .
$$

Now if $\sigma \in H^{0}\left(I_{k+1}, \pi_{k+1}^{*} L_{k+1}\right)$, by hypothesis H1, $j^{\prime *} \sigma=\pi^{\prime *} \alpha$ for some $\alpha \in H^{0}\left(Z,\left(\pi_{k+1} \circ j\right)^{*} L_{k+1}\right)$. We show now that $j^{*} \sigma=\alpha$. Indeed, by property $\mathrm{H} 2$, it suffices to show that this is true after restriction to $Z_{1}$, and since $\phi: Z_{1}^{\prime} \rightarrow Z_{1}$ is dominating, it suffices to show that

$$
\phi^{*}\left(\alpha_{\mid Z_{1}}\right)=\phi^{*}\left(j^{*} \sigma_{\mid Z_{1}}\right) .
$$

But this follows from $j \circ \phi=j_{\mid Z_{1}^{\prime}}^{\prime}$ and from $j^{\prime *} \sigma=\pi^{\prime *} \alpha$, with $\phi=\pi_{\mid Z_{1}^{\prime}}^{\prime}$

Finally it follows from the equalities $\alpha=j^{*} \sigma$ and $j^{\prime *} \sigma=\pi^{\prime *} \alpha$ that

$$
\sigma^{\prime}=\sigma-\pi^{*}\left(\frac{1}{k+1} \operatorname{Tr} \sigma\right)
$$

vanishes along $j(Z) \cup j^{\prime}\left(Z^{\prime}\right)$. (Indeed, because we know that $j(Z)$ is not contained in the ramification locus of $\pi_{k+1}$ and $\pi_{k+1} \circ j: Z \rightarrow X_{\text {curv }}^{[k+1]}$ is generically one to one on its image, the map $\pi_{k+1}$ restricted to $j(Z) \cup j^{\prime}\left(Z^{\prime}\right)$ is generically a degree $k+1$ unramified map onto its image. On the other hand, the two equalities above say that $\sigma_{\mid j(Z) \cup j^{\prime}\left(Z^{\prime}\right)}$ is generically a pull-back of a section of $L_{k+1}$ on this image. Hence the previously used trace argument applies to an open set of this image.)

Now this implies that $\sigma^{\prime}$ vanishes by hypothesis H3. This concludes the proof of our claim.

We conclude this section with the description of the schemes $Z, Z^{\prime}$ we will be considering.

Recall from [8], [11], [12], that there is a unique stable bundle $E$ of rank 2 on $S$, (the Lazarsfeld-Mukai vector bundle,) which satisfies the following properties:

$$
\operatorname{det} E=L, c_{2}(E)=k+1, h^{0}(E)=k+2 .
$$


Such vector bundle is obtained by choosing a line bundle $D$ on a generic member $C$ of $|L|$, such that $h^{0}(D)=2$ and $\operatorname{deg} D=k+1$. Such a line bundle exists by Brill-Noether theory, and it is generated by global sections since $C$ does not carry a $g_{k}^{1}$ by Lazarsfeld [11]. Then we have a vector bundle $F$ on $S$ defined by the exact sequence

$$
0 \rightarrow F \rightarrow H^{0}(D) \otimes \mathcal{O}_{S} \rightarrow D \rightarrow 0
$$

and $E$ is defined as the dual of $F$. The stability of $E$ follows from the fact that Pic $S=\mathbb{Z} L$ and $H^{0}(S, E(-L))=0$. The uniqueness of such $E$ follows then from the fact that $\chi\left(E, E^{\prime}\right)=2$ for any other vector bundle $E^{\prime}$ with the same numerical properties, so that either $\operatorname{Hom}\left(E, E^{\prime}\right) \neq 0$ or $\operatorname{Hom}\left(E^{\prime}, E\right) \neq 0$. But then by stability, $E=E^{\prime}$.

The property $h^{0}(S, E)=k+2$ follows from the sequence dual to (2.12)

$$
0 \rightarrow H^{0}(D)^{*} \otimes \mathcal{O}_{S} \rightarrow E \rightarrow K_{C}-D \rightarrow 0,
$$

and from Riemann-Roch which gives $h^{0}\left(K_{C}-D\right)=k$.

Another way to construct the bundle $E$ is via Serre's construction. By RiemannRoch the divisors $D$ of degree $k+1$ on smooth members $C$ of $|L|$ which satisfy $h^{0}(C, D)=2$ are exactly the subschemes $z$ of degree $k+1$ on $S$ contained in a smooth member $C$ of $|L|$ and satisfying the condition that the restriction map

$$
H^{0}(S, L) \rightarrow H^{0}\left(L_{\mid z}\right)
$$

is not surjective. Note that since the curves $C$ are general in the sense of BrillNoether, the corank of this map is exactly 1 and furthermore for any $z^{\prime} \subsetneq z$ the restriction map

$$
H^{0}(S, L) \rightarrow H^{0}\left(L_{\mid z^{\prime}}\right)
$$

is surjective. Hence, since $K_{S}$ is trivial, to such $z$ corresponds a vector bundle $E$ together with a section $\sigma_{z}$ vanishing on $z$. This $E$ is an extension

$$
0 \rightarrow \mathcal{O}_{S} \stackrel{\sigma_{z}}{\rightarrow} E \rightarrow \stackrel{\wedge \sigma_{z}}{\rightarrow} \mathcal{I}_{z}(L) \rightarrow 0
$$

Computing the numerical invariants of this bundle $E$, and arguing as before by stability, we see that this bundle is isomorphic to the one constructed above. Notice that each $g_{k+1}^{1}, D$ on a smooth member $C \in|L|$ provides by (2.13) a rank 2 subspace of sections of $E$, and that the zero sets of these sections identify to the members of $|D|$, as subschemes of $S$.

It follows from the exact sequence (2.14) twisted by $E$ that $h^{0}\left(S, E \otimes \mathcal{I}_{z}\right)=1$ for any $z$ as above. Hence the morphism

$$
\mathbb{P}\left(H^{0}(S, E)\right) \rightarrow S^{[k+1]}
$$

which to $\sigma$ associates its zero set, is in fact an embedding. One sees easily that the open set $\mathbb{P}\left(H^{0}(S, E)\right)_{\text {curv }}$ corresponding to curvilinear subschemes is large in $\mathbb{P}\left(H^{0}(S, E)\right)$. 
Let now $W:=\pi_{k+1}^{-1}\left(\mathbb{P}\left(H^{0}(S, E)\right)_{\text {curv }}\right) \subset I_{k+1} . W$ is easily shown to be smooth. There is a natural morphism

$$
\psi: W \rightarrow S_{\text {curv }}^{[k]}
$$

defined as the restriction of $p r_{2} \circ \tau$ to $W$. This $\psi$ can be shown to be generically of degree one on its image.

Consider the blow-up $\widetilde{S \times W}$ of $S \times W$ along $K:=(I d, \psi)^{-1}\left(I_{k}\right)$. It admits a morphism $\widetilde{(I d, \psi)}$ to the blow-up of $S \times S_{\text {curv }}^{[k]}$ along $I_{k}$, and the later contains $I_{k+1}$ as a large open set. One verifies that $\widetilde{I d, \psi}^{-1}\left(I_{k+1}\right)$ is a large open set of $\widetilde{S \times W}$. This will be our scheme $Z$. The morphism $j: Z \rightarrow I_{k+1}$ will be simply the restriction to $Z$ of $\widetilde{(d d, \psi)}$.

Again one can show (using now the assumption that $k>1$ ) that the morphism $\pi_{k+1} \circ j: Z \rightarrow S^{[k+1]}$ is generically of degree one on its image.

Next let $\pi^{\prime \prime}: \tilde{W} \rightarrow W$ be the degree $k$ cover obtained by completing the Cartesian diagram

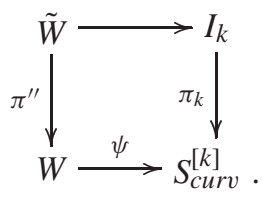

Consider the rational map

$$
j^{\prime}: S \times \tilde{W}---->I_{k+1}
$$

which to $\left(s, s_{1}, w\right), s_{1} \in \operatorname{Supp} \psi(w)$ associates $\left(s_{1}, s \cup \psi(w)\right)$. This morphism becomes well defined after blowing-up $K^{\prime}:=\left(I d, \pi^{\prime \prime}\right)^{-1}(K)$ and restricting to a large open subset. Our scheme $Z^{\prime}$ will be this large open set. The morphism $\pi^{\prime}: Z^{\prime} \rightarrow Z$ is the restriction to $Z^{\prime}$ of the morphism $B l_{K^{\prime}}(S \times \tilde{W}) \rightarrow B l_{K}(S \times Z)$ induced by $\left(I d, \pi^{\prime \prime}\right)$. The morphism $j^{\prime}: Z^{\prime} \rightarrow I_{k+1}$ is induced by the rational map $j^{\prime}$ above. We have

$$
\pi_{k+1} \circ j^{\prime}=\pi_{k+1} \circ j \circ \pi^{\prime} .
$$

Indeed, both maps send $\left(s, s_{1}, w\right), s_{1} \in \operatorname{Supp} \psi(w)$ to $s \cup \psi(w)$. It is obvious that $\pi_{k+1}^{-1}\left(\pi_{k+1} \circ j(Z)\right)$ is equal to $j(Z) \cup j^{\prime}\left(Z^{\prime}\right)$. Indeed, the fiber over $s \cup \psi(w) \in$ $\pi_{k+1} \circ j(Z)$ consists in choosing one point in the scheme $s \cup \psi(w)$. This point may be $s$, in which case we are in $j(Z)$, or has to be a point $s_{1}$ contained in $\operatorname{Supp} \psi(w)$ in which case it determines a point $\left(s_{1}, w\right)$ of $\tilde{W}$ over $w$, and we are then in $j^{\prime}\left(Z^{\prime}\right)$.

Remark 2. The scheme $Z$ is non necessarily smooth, but one can show that $K$ is reduced, so that its singular locus is of codimension at least two in $S \times W$. The same thing is true for $Z^{\prime}$ and $K^{\prime}$. If one wants to work with smooth schemes $Z_{0}$ and $Z_{0}^{\prime}$ (so as to be exactly in the conditions (H) described above), it suffices to restrict to the blowing-ups of $S \times W-K_{\text {sing }}$ along $K-K_{\text {sing }}$ and $S \times W-K_{\text {sing }}^{\prime}$ along $K^{\prime}-K_{\text {sing }}^{\prime}$. All what follows will be true for these subschemes. 
To conclude, it remains now to construct $Z_{1}$ and $Z_{1}^{\prime}$. $Z_{1}$ will be the exceptional divisor of $Z$ (recall that $Z$ is a large open set in $B l_{K}(S \times W)$ ). Hence $Z_{1}$ is the inverse image under the blow-up map $Z \rightarrow S \times W$ of

$$
K=\{(s, w) \in S \times W, s \in \operatorname{Supp} \psi(w)\} .
$$

We now construct a generic lifting of $Z_{1}$ in $Z^{\prime}$, the closure of the image of which will be $Z_{1}^{\prime}$. By definition of $Z^{\prime}$ as a large open set of $B l_{K^{\prime}}(S \times \tilde{W})$, it suffices to construct a lifting of $K$ to a component of $K^{\prime}$ in $S \times \tilde{W}$. But if $(s, w) \in K$, we have that $s \in \operatorname{Supp} \psi(w)$ so that $(s, w)$ identifies to an element $\tilde{w}$ of $\tilde{W}$. Our lifting sends simply $(s, w)$ to $(s, \tilde{w})$.

It remains finally to see that the morphisms $j^{\prime}$ and $j \circ \pi^{\prime}$ agree on $Z_{1}^{\prime}$. Since $I_{k+1}$ is contained in $S \times S_{\text {curv }}^{[k+1]}$, it suffices to prove that $p r_{1} \circ j^{\prime}$ and $p r_{1} \circ j \circ \pi^{\prime}$ agree on $Z_{1}^{\prime}$ and that $p r_{2} \circ j^{\prime}$ and $p r_{2} \circ j \circ \pi^{\prime}$ agree on $Z_{1}^{\prime}$, with $p r_{2}=\pi_{k+1}$ on $I_{k+1}$. For the first one this is obvious since both maps factor through the contraction $Z_{1}^{\prime} \rightarrow K^{\prime}$, and are equal on $K^{\prime} \subset S \times \tilde{W}$ to the first projection on $S$, as follows from the definition of the lifting $K \rightarrow K^{\prime}$.

As for the second one, it follows from the fact that, by construction, $\pi_{k+1} \circ j^{\prime}$ and $\pi_{k+1} \circ j \circ \pi^{\prime}$ agree on $Z^{\prime}$.

\section{Proof of the assumptions $\mathrm{H} 2$ and $\mathrm{H3}$}

We start with the proof of hypothesis H2.

Proposition 1. Let

$$
Z_{1} \subset Z \stackrel{\pi_{k+1} \circ j}{\longrightarrow} S^{[k+1]}
$$

be as in the previous section. Then the restriction map

$$
H^{0}\left(Z,\left(\pi_{k+1} \circ j\right)^{*} L_{k+1}\right) \rightarrow H^{0}\left(Z_{1},\left(\pi_{k+1} \circ j\right)^{*} L_{k+1 \mid Z_{1}}\right)
$$

is injective.

The proof will be obtained by restricting the construction to a generic smooth member $C \in|L|$. Indeed, recall that $Z$ is a large open set in the blow-up of $S \times W$ along the incidence subscheme $K=(i d, \psi)^{-1}\left(I_{k}\right)$, where

$$
W=\left\{(x, \sigma) \in S \times \mathbb{P}\left(H^{0}(S, E)\right)_{c u r v}, \sigma(x)=0\right\},
$$

and $\psi: W \rightarrow S^{[k]}$ sends $(x, \sigma)$ to the residual scheme of $x$ in $V(\sigma)$. Now since $k \geq 1$, the generic element $z=V(\sigma)$ is supported in a pencil of elements of $|L|$, the generic member being smooth. It follows that a generic element of $S \times W$ is of the form $\left(s_{1}, s_{2}, z\right), z=V(\sigma), \sigma\left(s_{2}\right)=0$ and there exists a smooth member $C \in|L|$ such that $s_{1}, s_{2}, z$ are supported on $C$. Hence it suffices to prove the analogue of Proposition 1 with $Z$ replaced by $Z_{C}$, the proper transform of $C \times W_{C}$ in $Z \subset B l_{K}(S \times W)$, where

$$
W_{C}:=\left\{(c, \sigma) \in C \times \mathbb{P}\left(H^{0}(S, E)\right), \sigma(c)=0, V(\sigma) \subset C\right\},
$$

and $Z_{1}$ is replaced by $Z_{1, C}:=Z_{1} \cap Z_{C}$. 
Proposition 2. The restriction map

$$
H^{0}\left(Z_{C},\left(\pi_{k+1} \circ j\right)^{*} L_{k+1 \mid Z_{C}}\right) \rightarrow H^{0}\left(Z_{1},\left(\pi_{k+1} \circ j\right)^{*} L_{k+1 \mid Z_{1, C}}\right)
$$

is injective.

Proof. By the description of the bundle $E$ given in the previous section, we note that the set

$$
\left\{\sigma \in \mathbb{P}\left(H^{0}(S, E)\right), \quad V(\sigma) \subset C\right\},
$$

identifies by the map $\sigma \mapsto V(\sigma)$ to the disjoint union of the $\mathbb{P}^{1} \subset C^{(k+1)}$ corresponding to $g_{k+1}^{1}$,s on $C$. If $D$ is such a $g_{k+1}^{1}$ on $C, D$ gives a morphism of degree $k+1$

$$
\phi_{D}: C \rightarrow \mathbb{P}^{1}
$$

or a line bundle $L_{D}$ on $C$ of degree $k+1$ with two sections. By definition, $W_{C}$ identifies (via $\psi$ ) to the disjoint union of copies $C_{D}$ of $C$ contained in $C^{(k)}$ and indexed by the $g_{k+1}^{1}$ 's $D$ of $C$, where the map

$$
\psi_{D}: C \cong C_{D} \rightarrow C^{(k)}
$$

is given by

$$
c \mapsto \text { the unique effective divisor equivalent to } D-c \text {. }
$$

Finally $Z_{C}$ identifies to a disjoint union of surfaces $Z_{C, D}$ isomorphic to $C \times C$, since the pull-back $\Delta_{D}$ to $C \times C_{D}$ of the incidence scheme in $C \times C^{(k)}$ is of pure codimension 1, so that the blow-up of $C \times C_{D}$ along $\Delta_{D}$ is isomorphic to $C \times C_{D}$. Note that under this isomorphism, the intersection of $Z_{1}$ with $C \times C_{D}$ becomes identified to $\Delta_{D}$.

Recall now that on the large open set $Z \subset \widehat{S \times W}$, we have

$$
\left(\pi_{k+1} \circ j\right)^{*} L_{k+1}=\tau^{*}\left(L \otimes \psi^{*} L_{k}\right)\left(-Z_{1}\right) .
$$

We have $L_{\mid C}=K_{C}$ and in the sequel we will use the notation $H_{D}$ for the line bundle $L_{k \mid C_{D}}$. (It will be shown that $H_{D} \equiv k L_{D}$ but this will not be used now.) Restricting the equality above to $Z_{C}$, we have to show that for each $D$ the restriction map

$$
H^{0}\left(C \times C, K_{C} \otimes H_{D}\left(-\Delta_{D}\right)\right) \rightarrow H^{0}\left(\Delta_{D}, K_{C} \otimes H_{D}\left(-\Delta_{D}\right)_{\mid \Delta_{D}}\right)
$$

is injective. In other words we want to show that

$$
H^{0}\left(C \times C, K_{C} \otimes H_{D}\left(-2 \Delta_{D}\right)\right)=0 .
$$

Now, since $\Delta_{D}$ is the restriction to $C \times C_{D}$ of the incidence scheme, and since $C_{D}$ parametrizes the effective divisors of the form $L_{D}-x, x \in C$, it is clear that

$$
\Delta_{D}=\left(\phi_{D}, \phi_{D}\right)^{-1}\left(\operatorname{diag}\left(\mathbb{P}^{1}\right)\right)-\operatorname{diag}(C) \text {. }
$$

Hence we have

$$
\Delta_{D} \equiv L_{D} \otimes L_{D}-\operatorname{diag}(C)
$$


in $C \times C$. It follows that

$$
K_{C} \otimes H_{D}\left(-2 \Delta_{D}\right) \equiv\left(K_{C}-2 L_{D}\right) \otimes\left(H_{D}-2 L_{D}\right)+2 \operatorname{diag} C .
$$

Now we have the equality

$$
H^{0}\left(C, K_{C}-2 L_{D}\right)=0
$$

which is proven in [11], since $C$ is generic in $S$. (Indeed for a base point free pencil, $\left|L_{D}\right|$, the condition that the $\mu_{0}$-map

$$
H^{0}\left(C, L_{D}\right) \otimes H^{0}\left(C, K_{C}-L_{D}\right) \rightarrow H^{0}\left(C, K_{C}\right)
$$

is injective is equivalent by the base-point free pencil trick to the condition

$$
\left.H^{0}\left(C, K_{C}-2 L_{D}\right)=0 .\right)
$$

The equality (3.15) follows now from (3.16) and from the fact that the map $H^{0}\left(C, 2 L_{D}\right) \rightarrow H^{0}\left(2 L_{D \mid 2 x}\right)$ is surjective for generic $x$ in $C$. Hence by RiemannRoch, $H^{0}\left(C, K_{C}-2 L_{D}\right)=0$ implies $H^{0}\left(C, K_{C}-2 L_{D}+2 x\right)=0$ for generic $x \in C$. It follows that

$$
H^{0}\left(C \times C,\left(K_{C}-2 L_{D}\right) \otimes\left(H_{D}-2 L_{D}\right)+2 \operatorname{diag} C\right)=0,
$$

which proves the Proposition 2, and hence Proposition 1 is proven.

We turn now to the proof of hypothesis $\mathrm{H} 3$.

Proposition 3. The morphism $Z \stackrel{j}{\rightarrow} I_{k+1}$ being defined as in the previous section, the pull-back map

$$
j^{*}: H^{0}\left(I_{k+1}, \pi_{k+1}^{*} L_{k+1}\right) \rightarrow H^{0}\left(Z,\left(\pi_{k+1} \circ j\right)^{*} L_{k+1}\right)
$$

is injective.

The proof proceeds in several steps, and occupies the remainder of this section. Recall that $I_{k+1}$ is a large open set in the blow-up of $S \times S^{[k]}$ along the incidence subscheme $I_{k}$ and that we have the following formula

$$
\pi_{k+1}^{*} L_{k+1}=\tau^{*}\left(L \otimes L_{k}\right)(-D),
$$

where $D$ is the exceptional divisor and $\tau$ is the blowing-up map. Since $Z$ is a large open set in the proper transform of this blowing-up under the morphism $(I d, \psi): S \times W \rightarrow S \times S^{[k]}$, it suffices to prove

Proposition 4. The restriction map

$$
\psi^{*}: H^{0}\left(S^{[k]}, L_{k}\right) \rightarrow H^{0}\left(W, \psi^{*} L_{k}\right)
$$

is injective. 
In order to prove this proposition, we first show

Lemma 2. Denoting by $\pi: W \rightarrow \mathbb{P}\left(H^{0}(S, E)\right)$ the restriction of the morphism $\pi_{k+1}: I_{k+1} \rightarrow S^{[k+1]}$, we have the formula

$$
\psi^{*} L_{k}=\pi^{*} \mathcal{O}_{\mathbb{P}\left(H^{0}(S, E)\right)}(k) .
$$

Proof. By definition, $\psi^{*} L_{k}=\operatorname{det} \psi^{*} \mathcal{E}_{L, k}$, where the bundle $\mathcal{E}_{L, k}$ has for fiber $H^{0}\left(L_{\mid z}\right)$ at a point $z \in S^{[k]}$. Now, if $z \in W$, the scheme $z^{\prime}=\psi(z)$ has length $k$, hence the restriction map

$$
H^{0}(S, L) \rightarrow H^{0}\left(L_{\mid z^{\prime}}\right)
$$

is surjective. On the other hand if $z^{\prime \prime}=\pi(z)$, we have $z^{\prime} \subset z^{\prime \prime}$ and the restriction map

$$
H^{0}(S, L) \rightarrow H^{0}\left(L_{\mid z^{\prime \prime}}\right)
$$

is not surjective. Hence we have

$$
H^{0}\left(S, L \otimes \mathcal{I}_{z^{\prime}}\right)=H^{0}\left(S, L \otimes \mathcal{I}_{z^{\prime \prime}}\right),
$$

and the fiber of $\psi^{*} \mathcal{E}_{L, k}$ at $z$ is canonically isomorphic to $H^{0}(S, L) / H^{0}\left(S, L \otimes \mathcal{I}_{\pi(z)}\right)$. Hence we have

$$
\psi^{*} L_{k}=-\pi^{*} \operatorname{det} \mathcal{F},
$$

where the bundle $\mathcal{F}$ on $\mathbb{P}\left(H^{0}(S, E)\right)$ is the bundle with fiber $H^{0}\left(S, L \otimes \mathcal{I}_{z_{\sigma}}\right)$ at $\sigma, z_{\sigma}=V(\sigma)$. Now recall that for each $\sigma$ we have the exact sequence

$$
0 \rightarrow \mathcal{O}_{S} \stackrel{\sigma}{\rightarrow} E \stackrel{\wedge \sigma}{\rightarrow} \mathcal{I}_{z_{\sigma}}(L) \rightarrow 0
$$

This induces the exact sequence

$$
0 \rightarrow<\sigma>\rightarrow H^{0}(S, E) \stackrel{\wedge \sigma}{\rightarrow} H^{0}\left(S, \mathcal{I}_{z_{\sigma}}(L)\right) \rightarrow 0 .
$$

We conclude immediately from this that $\mathcal{F}$ fits into the exact sequence

$$
0 \rightarrow \mathcal{O}_{\mathbb{P}\left(H^{0}(S, E)\right)}(-2) \rightarrow H^{0}(S, E) \otimes \mathcal{O}_{\mathbb{P}\left(H^{0}(S, E)\right)}(-1) \rightarrow \mathcal{F} \rightarrow 0 .
$$

Since rank $H^{0}(S, E)=k+2$, it follows that det $\mathcal{F}=\mathcal{O}_{\mathbb{P}\left(H^{0}(S, E)\right)}(-k)$.

It follows from this lemma that we have a natural inclusion

$$
S^{k} H^{0}(S, E)^{*} \hookrightarrow H^{0}\left(W, \psi^{*} L_{k}\right) .
$$

(It will be proven in the next section that this inclusion is in fact an isomorphism, but we shall not need this here.)

Our strategy to prove Proposition 4 will be first to construct an isomorphism

$$
H^{0}\left(S^{[k]}, L_{k}\right)=\wedge^{k} H^{0}(S, L) \cong S^{k} H^{0}(S, E)^{*}
$$

and then to show that composed with the inclusion (3.17), it is equal, up to a coefficient, to the pull-back map $\psi^{*}$. 
Construction of the isomorphism (3.18). We note first that the determinant map

$$
\operatorname{det}: \bigwedge^{2} H^{0}(S, E) \rightarrow H^{0}(S, \operatorname{det} E)=H^{0}(S, L)
$$

does not vanish on any element of rank 2. Indeed, such an element of rank 2 is given by a subspace $W$ of rank 2 of $H^{0}(S, E)$, and if its determinant would vanish this would imply that $W$ generates a rank 1 subsheaf of $E$ with at least two sections. But since Pic $S$ is generated by $L$ and $H^{0}(S, E(-L))=0$ this is impossible. Hence det provides a morphism

$$
d: G_{2} \rightarrow \mathbb{P}\left(H^{0}(S, L)\right),
$$

where $G_{2}$ is the Grassmannian of rank two vector subspaces of $H^{0}(S, E)$, or dually a base-point free linear system

$$
K:=H^{0}(S, L)^{*} \stackrel{d^{*}}{\rightarrow} H^{0}\left(G_{2}, \mathcal{L}\right)=\wedge^{2} H^{0}(S, E)^{*},
$$

where $\mathcal{L}$ is the Plücker polarization on $G_{2}$. Notice that since rank $K=2 k+1$, and $\operatorname{dim} G_{2}=2 k, d^{*}$ has to be injective. Since $K$ is base-point free, we have the exact Koszul complex on $G_{2}$

$$
0 \rightarrow \bigwedge^{2 k+1} K \otimes \mathcal{L}^{-(2 k+1)} \rightarrow \ldots \rightarrow K \otimes \mathcal{L}^{-1} \rightarrow \mathcal{O}_{G_{2}} \rightarrow 0
$$

We can now tensor this sequence with $S^{k} \mathcal{E}$, where the rank 2 vector bundle $\mathcal{E}$ on $G_{2}$ is dual to the tautological rank two subbundle and satisfies $H^{0}\left(G_{2}, S^{k} \mathcal{E}\right)=$ $S^{k} H^{0}(S, E)^{*}$.

This provides the exact complex

$$
0 \rightarrow \bigwedge^{2 k+1} K \otimes \mathcal{L}^{-(2 k+1)} \otimes S^{k} \mathcal{E} \rightarrow \ldots \rightarrow K \otimes \mathcal{L}^{-1} \otimes S^{k} \mathcal{E} \rightarrow S^{k} \mathcal{E} \rightarrow 0 .
$$

In this complex $\mathcal{K}$, the term $S^{k} \mathcal{E}$ is put in degree 0 . The hypercohomology $\mathbb{H}^{0}\left(G_{2}, \mathcal{K}\right)$ vanishes. Now we have a spectral sequence

$$
E_{1}^{p, q}=H^{q}\left(G_{2}, \mathcal{K}^{p}\right) \Rightarrow \mathbb{H}^{p+q}\left(G_{2}, \mathcal{K}^{\cdot}\right) .
$$

It is obvious for degree reasons that all differentials $d_{r}$ starting from the term $E_{r}^{0,0}$ vanish. On the other hand the terms $E_{1}^{p, q}$ with $p+q=-1$ are of the form

$$
H^{q}\left(G_{2}, \bigwedge^{q+1} K \otimes \mathcal{L}^{-q-1} \otimes S^{k} \mathcal{E}\right)
$$

Using the Proposition 9 proven in the appendix, we see that these terms are all 0 , except for

$$
E_{1}^{-k-1, k}=H^{k}\left(G_{2}, \bigwedge^{k+1} K \otimes \mathcal{L}^{-k-1} \otimes S^{k} \mathcal{E}\right)
$$


which is equal to $\bigwedge^{k+1} K$. It follows that there is only one non zero differential which arrives in some $E_{r}^{0,0}$, namely

$$
d_{k+1}: E_{k+1}^{-k-1, k} \rightarrow E_{k+1}^{0,0} .
$$

This implies that

$$
E_{k+1}^{0,0}=E_{1}^{0,0}=H^{0}\left(G_{2}, S^{k} \mathcal{E}\right)=S^{k} H^{0}(S, E)^{*}
$$

and that the differential $d_{k+1}$ above is surjective, since the spectral sequence abuts to 0 . Hence we have build a surjective map $d_{k+1}$ from a subquotient of $E_{1}^{-k-1, k}=$ $\bigwedge^{k+1} K$ to $S^{k} H^{0}(S, E)^{*}$. Since

$$
\operatorname{dim} \bigwedge^{k+1} K=\operatorname{dim} S^{k} H^{0}(S, E)^{*}=C_{2 k+1}^{k+1}
$$

this subquotient must in fact be equal to $\bigwedge^{k+1} K$ and the map $d_{k+1}$ has to be an isomorphism. Finally, since $\operatorname{rank} K=2 k+1$,

$$
\bigwedge^{k+1} K=\left(\bigwedge^{k} K\right)^{*}=\bigwedge^{k} H^{0}(S, L) .
$$

Hence we have constructed our isomorphism

$$
d_{k+1}: \bigwedge^{k} H^{0}(S, L) \rightarrow S^{k} H^{0}(S, E)^{*} .
$$

To conclude the proof of Proposition 4, it remains only to show:

Proposition 5. The map $d_{k+1}$ constructed above identifies up to a coefficient to the map

$$
\psi^{*}: H^{0}\left(S^{[k]}, L_{k}\right) \cong \bigwedge^{k} H^{0}(S, L) \rightarrow H^{0}\left(W, \psi^{*} L_{k}\right)
$$

which takes values in $S^{k} H^{0}(S, E)^{*} \subset H^{0}\left(W, \psi^{*} L_{k}\right)$.

Proof. First of all it is clear that $\psi^{*}$ takes values in $\pi^{*} H^{0}\left(\mathbb{P}\left(H^{0}(S, E)\right), \mathcal{O}(k)\right)=$ $S^{k} H^{0}(S, E)^{*}$. Indeed, this map is the pull-back map associated to the morphism

$$
\begin{gathered}
W \rightarrow \operatorname{Grass}\left(k+1, H^{0}(S, L)\right) \\
z \mapsto H^{0}\left(S, L \otimes \mathcal{I}_{z^{\prime}}\right), z^{\prime}=\psi(z) .
\end{gathered}
$$

But as mentioned in the proof of Lemma 2, this morphism factors through $\pi$ : $W \rightarrow \mathbb{P}\left(H^{0}(S, E)\right)$. More precisely, we noticed in the proof of Lemma 2 that the restriction map

$$
\psi^{*}: \wedge^{k} H^{0}(S, L) \rightarrow S^{k} H^{0}(S, E)^{*}
$$

corresponds to the morphism

$$
\mathbb{P}\left(H^{0}(S, E)\right) \rightarrow \operatorname{Grass}\left(k+1, H^{0}(S, L)\right)
$$




$$
\sigma \mapsto \operatorname{det}\left(\sigma \wedge H^{0}(S, E)\right)
$$

But this morphism is the composition of the morphism

$$
\begin{aligned}
\beta: \mathbb{P}\left(H^{0}(S, E)\right) & \rightarrow \operatorname{Grass}\left(k+1, \bigwedge^{2} H^{0}(S, E)\right) \\
\sigma & \mapsto \sigma \wedge H^{0}(S, E) .
\end{aligned}
$$

and of the rational map

$$
\operatorname{det}: \operatorname{Grass}\left(k+1, \bigwedge^{2} H^{0}(S, E)\right) \rightarrow \operatorname{Grass}\left(k+1, H^{0}(S, L)\right)
$$

induced by the determinant map det $: \bigwedge^{2} H^{0}(S, E) \rightarrow H^{0}(S, L)$.

Next, we note that, with the same spectral sequence argument, and replacing $K=H^{0}(S, L)^{*} \subset \bigwedge^{2} H^{0}(S, E)^{*}$ by the base point free linear system $K^{\prime}=$ $\bigwedge^{2} H^{0}(S, E)^{*}$ on $G_{2}$, we could have constructed more generally a surjective map

$$
D_{k+1}: \bigwedge^{k+1}\left(\bigwedge^{2} H^{0}(S, E)^{*}\right) \rightarrow S^{k} H^{0}(S, E)^{*},
$$

whose restriction to $\bigwedge^{k+1} K$ is equal to $d_{k+1}$.

Hence Proposition 5 will follow from the following

Lemma 3. The maps $D_{k+1}$ and $\beta^{*}$ from $\bigwedge^{k+1}\left(\bigwedge^{2} H^{0}(S, E)^{*}\right)$ to $S^{k} H^{0}(S, E)^{*}$ coincide up to a coefficient.

Proof. We could argue by $S l(k+2)$-equivariance. A more direct way to prove this is to note the following: If $W \subset \bigwedge^{2} H^{0}(S, E)^{*}$ is a rank $k+1$ vector subspace in general position, it defines a codimension $k+1$ complete intersection subvariety $G_{W}$ of $G_{2}$. Consider the incidence correspondence

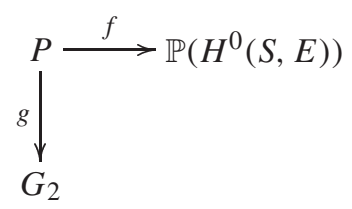

Then we have a hypersurface $X_{W}=f\left(g^{-1}\right)\left(G_{W}\right)$ of $\mathbb{P}\left(H^{0}(S, E)\right)$, which is easily proven to be of degree $k$. It is clear that

$$
H^{0}\left(G_{2}, S^{k} \mathcal{E} \otimes \mathcal{I}_{G_{W}}\right)=H^{0}\left(\mathbb{P}\left(H^{0}(S, E)\right), \mathcal{O}_{\mathbb{P}\left(H^{0}(S, E)\right)}(k)\left(-X_{W}\right)\right) .
$$

On the other hand, from the linear system $W$ we can construct a Koszul complex which is a resolution of $\mathcal{I}_{G_{W}}$. Hence it is clear that

$$
D_{k+1}\left(\bigwedge^{k+1} W\right) \subset H^{0}\left(G_{2}, S^{k} \mathcal{E} \otimes \mathcal{I}_{G_{W}}\right)
$$


In other words, if $\eta$ is a generator of $\bigwedge^{k+1} W, D_{k+1}(\eta)$ is a defining equation of $X_{W}$ or 0 . It remains then only to prove that $\beta^{*} \eta$ also vanishes on $X_{W}$. But by definition

$$
X_{W}=\left\{x \in \mathbb{P}\left(H^{0}(S, E)\right), \exists 0 \neq \gamma \in \mathbb{P}\left(H^{0}(S, E) /<x>\right), x \wedge \gamma \perp W\right\} .
$$

This means that for $x \in X_{W}$, the composed map

$$
W \hookrightarrow \bigwedge^{2} H^{0}(S, E)^{*} \rightarrow\left(x \wedge H^{0}(S, E)\right)^{*}
$$

is not an isomorphism, hence its determinant vanishes. But this determinant is equal to $\beta^{*} \eta(x)$.

\section{Proof of the assumption H1}

Recall that we have a Cartesian diagram

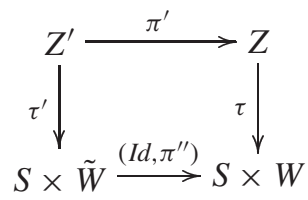

where the vertical maps $\tau, \tau^{\prime}$ are blow-ups and the degree $k$ morphism $\pi^{\prime \prime}$ fits into the Cartesian diagram

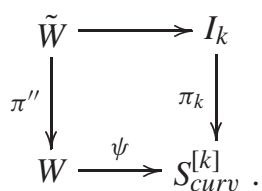

We have the morphisms

$$
j^{\prime}: Z^{\prime} \rightarrow I_{k+1}, j: Z \rightarrow I_{k+1}
$$

such that $\pi_{k+1} \circ j^{\prime}=\pi_{k+1} \circ j \circ \pi^{\prime}$ and the formula

$$
\left(\pi_{k+1} \circ j\right)^{*} L_{k+1}=\tau^{*}\left(L \otimes \psi^{*} L_{k}\right)(-D)
$$

where $D$ is the exceptional divisor of $\tau$. Pulling-back this equality to $Z^{\prime}$, we have

$$
\left(\pi_{k+1} \circ j^{\prime}\right)^{*} L_{k+1}=\tau^{\prime *}\left(L \otimes\left(\psi \circ \pi^{\prime \prime}\right)^{*} L_{k}\right)\left(-D^{\prime}\right),
$$

where $D^{\prime}$ is the exceptional divisor of $\tau^{\prime}$. Since $D^{\prime}=\pi^{\prime-1}(D)$ and $\pi^{\prime}$ is surjective, we conclude that in order to prove $\mathrm{H} 1$, that is the fact that the pull-back map

$$
\pi^{\prime *}: H^{0}\left(Z,\left(\pi_{k+1} \circ j\right)^{*} L_{k+1}\right) \rightarrow H^{0}\left(Z^{\prime},\left(\pi_{k+1} \circ j^{\prime}\right)^{*} L_{k+1}\right)
$$

is surjective, it suffices to show that the pull-back map

$$
\pi^{\prime \prime *}: H^{0}\left(W, \psi^{*} L_{k}\right) \rightarrow H^{0}\left(\tilde{W},\left(\psi \circ \pi^{\prime \prime}\right)^{*} L_{k}\right)
$$

is surjective. 
Now recall that we have a morphism

$$
\pi: W \rightarrow \mathbb{P}\left(H^{0}(S, E)\right)
$$

such that (cf Lemma 2)

$$
\psi^{*} L_{k}=\pi^{*} \mathcal{O}_{\mathbb{P}\left(H^{0}(S, E)\right)}(k) .
$$

Denoting by $r:=\pi \circ \pi^{\prime \prime}: \tilde{W} \rightarrow \mathbb{P}\left(\left(H^{0}(S, E)\right)\right.$, we shall prove the following stronger statement

Theorem 2. The pull-back map

$$
r^{*}: H^{0}\left(\mathbb{P}\left(H^{0}(E)\right), \mathcal{O}_{\mathbb{P}\left(H^{0}(E)\right)}(k)\right) \rightarrow H^{0}\left(\tilde{W},\left(\psi \circ \pi^{\prime \prime}\right)^{*} L_{k}\right)
$$

is surjective.

The end of this section will be devoted to the proof of this theorem, which proceeds in several steps. In what follows, we shall use the notation $H^{0}(E)$ for $H^{0}(S, E)$.

Notice to begin with that $\tilde{W}$ is a large open set in the subscheme

$$
W^{\prime} \subset \widetilde{S \times S} \times \mathbb{P}\left(H^{0}(E)\right),
$$

where $\widetilde{S \times S}$ is the blow-up of $S \times S$ along the diagonal, defined as

$$
W^{\prime}:=\left\{(x, y, \eta, \sigma), \sigma_{\mid \eta=0},\{x\},\{y\} \subset \eta\right\} .
$$

(Here $\eta$ is a subscheme of length 2 of $S$, and we see elements of $\widetilde{S \times S}$ as elements $(x, y)$ of $S \times S$ together with a schematic structure $\eta$ of length 2 on $\{x\} \cup\{y\}$.)

The map $r$ is just the restriction to $W^{\prime}$ of the second projection. Hence we have

$$
H^{0}\left(\tilde{W},\left(\psi \circ \pi^{\prime \prime}\right)^{*} L_{k}\right)=H^{0}\left(W^{\prime}, p r_{2}^{*} \mathcal{O}_{\mathbb{P}\left(H^{0}(E)\right)}(k)\right)
$$

and the surjectivity of (4.20) is equivalent to the condition

$$
H^{1}\left(\widetilde{S \times S} \times \mathbb{P}\left(H^{0}(E)\right), p r_{2}^{*} \mathcal{O}(k) \otimes \mathcal{I}_{W^{\prime}}\right)=0 .
$$

Now notice that there is a vector bundle $\tilde{E}_{2}$ on $\widetilde{S \times S}$ such that $W^{\prime}$ is the zero set of a section $\sigma$ of $\tilde{E}_{2} \otimes \mathcal{O}_{\mathbb{P}\left(H^{0}(E)\right)}(1)$. Indeed it suffices to take for $\tilde{E}_{2}$ the vector bundle with fiber $H^{0}\left(E_{\mid \eta}\right)$ at the point $(x, y, \eta)$ of $\widetilde{S \times S}$. Then the section $\sigma$ takes the value $\tau_{\mid \eta}$ at the point $(x, y, \eta, \tau)$ of $\widehat{S \times S} \times \mathbb{P}\left(H^{0}(E)\right)$. One checks easily that $W^{\prime}$ is reduced of codimension 4 . Hence we have a Koszul resolution of $\mathcal{I}_{W^{\prime}}$

$$
0 \rightarrow \bigwedge^{4} \tilde{E}_{2}^{*} \otimes \mathcal{O}(-4) \rightarrow \ldots \rightarrow \tilde{E}_{2}^{*} \otimes \mathcal{O}(-1) \rightarrow \mathcal{I}_{W^{\prime}} \rightarrow 0
$$

Our first goal will be to compute the cohomology groups of $\widetilde{S \times S} \times \mathbb{P}\left(H^{0}(E)\right)$ with value in $\bigwedge^{i} \tilde{E}_{2}^{*} \otimes \mathcal{O}(k-i)$. Since $k \geq 2, i \leq 4, \mathcal{O}(k-i)$ has no higher cohomology on $\mathbb{P}\left(H^{0}(E)\right)=\mathbb{P}^{k+1}$. Hence we have

$H^{l}\left(\widetilde{S \times S} \times \mathbb{P}\left(H^{0}(E)\right), \bigwedge^{i} \tilde{E}_{2}^{*} \otimes \mathcal{O}(k-i)\right)=H^{l}\left(\widetilde{S \times S}, \bigwedge^{i} \tilde{E}_{2}^{*}\right) \otimes S^{k-i} H^{0}(S, E)^{*}$.

We have now the following proposition 
Proposition 6. 1. $H^{2}\left(\widetilde{S \times S}, \tilde{E}_{2}^{*}\right)=p r_{1}^{*} H^{2}\left(S, E^{*}\right) \oplus p r_{2}^{*} H^{2}\left(S, E^{*}\right)$ and

$$
H^{1}\left(\widetilde{S \times S}, \tilde{E}_{2}^{*}\right)=0 .
$$

2. $H^{2}\left(\widetilde{S \times S}, \bigwedge^{2} \tilde{E}_{2}^{*}\right)=p r_{1}^{*} H^{2}(S,-L) \oplus p r_{2}^{*} H^{2}(S,-L)$.

3. $H^{4}\left(\widetilde{S \times S}, \bigwedge^{4} \tilde{E}_{2}^{*}\right)$ is dual to $\operatorname{Ker}\left(H^{0}(S, L) \otimes H^{0}(S, L) \rightarrow H^{0}(S, 2 L)\right)$.

4. $H^{3}\left(\widetilde{S \times S}, \bigwedge^{3} \tilde{E}_{2}^{*}\right)=0$ and $H^{4}\left(\widetilde{S \times S}, \bigwedge^{3} \tilde{E}_{2}^{*}\right)$ admits as a quotient

$$
\left.H^{4} \widetilde{(S \times S}, \tau^{*}\left(E^{*} \otimes(-L)\right)(2 \Delta)\right) \oplus H^{4}\left(\widetilde{S \times S}, \tau^{*}\left((-L) \otimes E^{*}\right)(2 \Delta)\right),
$$

which is dual to the direct sum of two copies of

$$
\operatorname{Ker}\left(H^{0}(S, E) \otimes H^{0}(S, L) \rightarrow H^{0}(S, E \otimes L)\right) .
$$

(Here $\Delta \subset \widetilde{S \times S}$ is the exceptional divisor.)

Proof. 1. The bundle $\tilde{E}_{2}$ fits into the exact sequence

$$
0 \rightarrow \tilde{E}_{2} \rightarrow \tau^{*}\left(p r_{1}^{*} E \oplus p r_{2}^{*} E\right) \rightarrow \tau^{\prime *} E \rightarrow 0
$$

where $\tau: \widetilde{S \times S} \rightarrow S \times S$ is the blowing-down map, and where $\tau^{\prime}: \Delta \rightarrow$ Diag $S$ is its restriction to the exceptional divisor.

Dualizing, we get the following exact sequence

$$
0 \rightarrow \tau^{*}\left(p r_{1}^{*} E^{*} \oplus p r_{2}^{*} E^{*}\right) \rightarrow \tilde{E}_{2}^{*} \rightarrow \tau^{\prime *} E^{*} \otimes \mathcal{O}_{\Delta}(\Delta) \rightarrow 0 .
$$

Now $R^{0} \tau_{*}^{\prime} \mathcal{O}_{\Delta}(\Delta)=R^{1} \tau_{*}^{\prime} \mathcal{O}_{\Delta}(\Delta)=0$ hence the sheaf on the right has no cohomology. It follows that

$$
\begin{gathered}
\left.H^{i}\left(\widetilde{S \times S}, \tilde{E}_{2}^{*}\right)=H^{i} \widetilde{(S \times S}, \tau^{*}\left(p r_{1}^{*} E^{*} \oplus p r_{2}^{*} E\right)\right) \\
=H^{i}\left(S \times S, p r_{1}^{*} E^{*} \oplus p r_{2}^{*} E\right) .
\end{gathered}
$$

Since $E^{*}$ has no odd dimensional cohomology, nor $\mathcal{O}_{S}$, it follows from Künneth formula that the same is true for $p r_{1}^{*} E^{*} \oplus p r_{2}^{*} E$ on $S \times S$. Finally we have

$$
H^{2}\left(S \times S, p r_{1}^{*} E^{*}\right)=H^{2}\left(S, E^{*}\right)
$$

since $H^{0}\left(S, E^{*}\right)=0$. This proves 1 .

2. From (4.24) we deduce that $\bigwedge^{2} \tilde{E}_{2}^{*}$ has a filtration whose successive terms are

$$
\begin{gathered}
\bigwedge^{2} \tau^{*}\left(p r_{1}^{*} E^{*} \oplus p r_{2}^{*} E^{*}\right), \quad \tau^{*}\left(p r_{1}^{*} E^{*} \oplus p r_{2}^{*} E^{*}\right) \otimes \tau^{\prime *} E^{*} \otimes \mathcal{O}_{\Delta}(\Delta), \\
\bigwedge^{2} \tau^{\prime *} E^{*} \otimes \mathcal{O}_{\Delta}(2 \Delta) .
\end{gathered}
$$


The sheaf $\left(p r_{1}^{*} E^{*} \oplus p r_{2}^{*} E^{*}\right) \otimes \tau^{\prime *} E^{*} \otimes \mathcal{O}_{\Delta}(\Delta)$ has no cohomology, since $\mathcal{O}_{\Delta}(\Delta)$ has no cohomology along the fibers of $\tau^{\prime}$. Hence we have an exact sequence

$$
\begin{aligned}
& H^{1}\left(\Delta, \bigwedge^{2} \tau^{\prime *} E^{*} \otimes \mathcal{O}_{\Delta}(2 \Delta)\right) \rightarrow H^{2}\left(\widetilde{S \times S}, \tau^{*} \bigwedge^{2}\left(p r_{1}^{*} E^{*} \oplus p r_{2}^{*} E^{*}\right)\right) \\
& \rightarrow H^{2}\left(\widetilde{S \times S}, \bigwedge^{2} \tilde{E}_{2}^{*}\right) \rightarrow H^{2}\left(\Delta, \bigwedge^{2} \tau^{\prime *} E^{*} \otimes \mathcal{O}_{\Delta}(2 \Delta)\right) \ldots
\end{aligned}
$$

But since

$$
R^{1} \tau_{*}^{\prime}\left(2 \Delta_{\mid \Delta}\right)=\mathcal{O}_{S}, R^{0} \tau_{*}^{\prime}\left(2 \Delta_{\mid \Delta}\right)=0,
$$

the term on the left is equal to $H^{0}\left(S, \bigwedge^{2} E^{*}\right)=0$ and the term on the right is equal to $H^{1}\left(S, \bigwedge^{2} E^{*}\right)=0$. Hence we have

$$
\begin{aligned}
H^{2}\left(\widetilde{S \times S}, \bigwedge^{2} \tilde{E}_{2}^{*}\right) & =H^{2}\left(\widetilde{S \times S}, \tau^{*} \bigwedge^{2}\left(p r_{1}^{*} E^{*} \oplus p r_{2}^{*} E^{*}\right)\right) \\
& =H^{2}\left(S \times S, \bigwedge\left(p r_{1}^{*} E^{*} \oplus p r_{2}^{*} E^{*}\right)\right)
\end{aligned}
$$

Finally

$$
\bigwedge^{2}\left(p r_{1}^{*} E^{*} \oplus p r_{2}^{*} E^{*}\right)=p r_{1}^{*} \bigwedge^{2} E^{*} \oplus E^{*} \otimes E^{*} \oplus p r_{2}^{*} \bigwedge^{2} E^{*}
$$

The central term has no cohomology in degree 2 by Künneth formula, because $H^{1}\left(S, E^{*}\right)=H^{0}\left(S, E^{*}\right)=0$, and we have

$$
H^{2}\left(S \times S, p r_{1}^{*} \bigwedge^{2} E^{*}\right)=H^{2}\left(S, \bigwedge^{2} E^{*}\right)=H^{2}(S,-L)
$$

where the first equality follows from Künneth formula and $H^{0}\left(S, \bigwedge^{2} E^{*}\right)=0$. This proves 2 .

3. We have $\operatorname{det} \tilde{E}_{2}^{*}=\tau^{*}((-L) \otimes(-L))(2 \Delta)$ by the exact sequence (4.23). Hence

$$
\bigwedge^{3} \tilde{E}_{2}^{*}=\tilde{E}_{2} \otimes \operatorname{det} \tilde{E}_{2}^{*}=\tilde{E}_{2} \otimes \tau^{*}((-L) \otimes(-L))(2 \Delta) .
$$

The exact sequence (4.23) gives now the long exact sequence

$$
\begin{gathered}
\left.H^{2}\left(\Delta, \tau^{\prime *}(E(-2 L))\left(2 \Delta_{\mid \Delta}\right)\right) \rightarrow H^{3} \widetilde{(\widetilde{S \times S}}, \bigwedge^{3} \tilde{E}_{2}^{*}\right) \\
\rightarrow H^{3}\left(\widetilde{S \times S}, \tau^{*}\left(\left(p r_{1}^{*} E \oplus p r_{2}^{*} E\right) \otimes((-L) \otimes(-L))\right)(2 \Delta)\right) .
\end{gathered}
$$

Since $R^{0} \tau_{*}^{\prime} \mathcal{O}_{\Delta}(2 \Delta)=0, R^{1} \tau_{*}^{\prime} \mathcal{O}_{\Delta}(2 \Delta)=\mathcal{O}_{S}$, the left hand side is equal to $H^{1}(S, E(-2 L))$, which is easily seen to be 0 .

Next we have $K_{\widetilde{S \times S}}=\mathcal{O}_{\widetilde{S \times S}}(\Delta)$, hence

$$
\left.H^{3}\left(\widetilde{S \times S}, \tau^{*}\left(p r_{1}^{*} E \oplus p r_{2}^{*} E\right) \otimes((-L) \otimes(-L))\right)(2 \Delta)\right)
$$


is dual to

$$
H^{1}\left(\widetilde{S \times S}, \tau^{*}\left(\left(p r_{1}^{*} E^{*} \oplus p r_{2}^{*} E^{*}\right) \otimes(L \otimes L)\right)(-\Delta)\right) .
$$

Writing the exact sequence

$0 \rightarrow \tau^{*}\left(\left(p r_{1}^{*} E^{*} \oplus p r_{2}^{*} E^{*}\right) \otimes(L \otimes L)\right)(-\Delta) \rightarrow \tau^{*}\left(\left(p r_{1}^{*} E^{*} \oplus p r_{2}^{*} E^{*}\right) \otimes(L \otimes L)\right)$

$$
\rightarrow \tau^{*}\left(\left(p r_{1}^{*} E^{*} \oplus p r_{2}^{*} E^{*}\right) \otimes(L \otimes L)\right)_{\mid \Delta} \rightarrow 0
$$

where

$$
\tau^{*}\left(\left(p r_{1}^{*} E^{*} \oplus p r_{2}^{*} E^{*}\right) \otimes(L \otimes L)\right)_{\mid \Delta} \cong \tau^{\prime *}\left(\left(E^{*} \oplus E^{*}\right)(2 L)\right),
$$

and using the isomorphism

$$
E^{*} \otimes L \cong E
$$

we see that the vanishing of the cohomology group (4.26) follows from the fact that the multiplication map

$$
H^{0}(S, E) \otimes H^{0}(S, L) \rightarrow H^{0}(S, E \otimes L)
$$

is surjective, which is easily checked, and from the vanishing

$$
H^{1}\left(S \times S,\left(p r_{1}^{*} E^{*} \oplus p r_{2}^{*} E^{*}\right) \otimes L \otimes L\right)=0 .
$$

Finally the equality (4.25) and the exact sequence (4.23) also show that $H^{4}\left(\widetilde{S \times S}, \wedge^{3} \tilde{E}_{2}^{*}\right)$ admits

$$
H^{4}\left(\widetilde{S \times S}, \tau^{*}\left(\left(p r_{1}^{*} E \oplus p r_{2}^{*} E\right) \otimes((-L) \otimes(-L))\right)(2 \Delta)\right)
$$

as a quotient. By Serre's duality this space is dual to

$$
H^{0}\left(\widetilde{S \times S}, \tau^{*}\left(\left(p r_{1}^{*} E^{*} \oplus p r_{2}^{*} E^{*}\right) \otimes(L \otimes L)\right)(-\Delta)\right) .
$$

But this is equal to

$$
H^{0}\left(S \times S,\left(p r_{1}^{*} E^{*} \oplus p r_{2}^{*} E^{*}\right) \otimes(L \otimes L) \otimes \mathcal{I}_{\text {Diag }}\right) .
$$

We use then the fact that

$$
p r_{1}^{*} E^{*} \otimes(L \otimes L)=E \otimes L
$$

to conclude that (4.27) is equal to the sum of two copies of

$$
\operatorname{Ker}\left(H^{0}(S, E) \otimes H^{0}(S, L) \rightarrow H^{0}(S, E \otimes L)\right) .
$$

4. We already noticed that

$$
\bigwedge^{4} \tilde{E}_{2}^{*}=\operatorname{det} \tilde{E}_{2}^{*}=\tau^{*}((-L) \otimes(-L))(2 \Delta) .
$$

It follows then from Serre's duality and $K_{\widetilde{S \times S}}=\mathcal{O}_{\widetilde{S \times S}}(\Delta)$ that $H^{4}\left(\widetilde{S \times S}, \wedge^{4} \tilde{E}_{2}^{*}\right)$ is dual to

$$
\left.H^{0}\left(\widetilde{S \times S}, \tau^{*}(L \otimes L)(-\Delta)\right)=\operatorname{Ker}\left(H^{0}(S, L) \otimes H^{0}(S, L) \rightarrow H^{0}(S, 2 L)\right)\right) .
$$

Hence 4 is proven. 
Coming back to the Koszul resolution of $\mathcal{I}_{W^{\prime}} \otimes p r_{2}^{*} \mathcal{O}(k)$ induced by (4.22), we see that in order to prove the vanishing (4.21), it suffices to show:

a) $H^{1}\left(\widetilde{S \times S} \times \mathbb{P}\left(H^{0}(E)\right), \tilde{E}_{2}^{*} \otimes \mathcal{O}(k-1)\right)=0$.

b) The interior product with $\sigma$

$$
\begin{aligned}
\operatorname{int}(\sigma): H^{2}\left(\widetilde{S \times S} \times \mathbb{P}\left(H^{0}(E)\right), \bigwedge^{2} \tilde{E}_{2}^{*} \otimes \mathcal{O}(k-2)\right) \\
\rightarrow H^{2}\left(\widetilde{S \times S} \times \mathbb{P}\left(H^{0}(E)\right), \tilde{E}_{2}^{*} \otimes \mathcal{O}(k-1)\right)
\end{aligned}
$$

is injective.

c) $H^{3}\left(\widetilde{S \times S} \times \mathbb{P}\left(H^{0}(E)\right), \bigwedge^{3} \tilde{E}_{2}^{*} \otimes \mathcal{O}(k-3)\right)=0$.

d) The interior product with $\sigma$

$$
\begin{aligned}
& \operatorname{int}(\sigma): H^{4}\left(\widetilde{S \times S} \times \mathbb{P}\left(H^{0}(E)\right), \bigwedge^{4} \tilde{E}_{2}^{*} \otimes \mathcal{O}(k-4)\right) \\
& \rightarrow H^{4}\left(\widetilde{S \times S} \times \mathbb{P}\left(H^{0}(E)\right), \bigwedge^{3} \tilde{E}_{2}^{*} \otimes \mathcal{O}(k-3)\right)
\end{aligned}
$$

is injective.

The conditions a) and c) have been established in Proposition 6. We now dualize property b) as follows: by Proposition 6, 1 and 2, we have

$$
\begin{aligned}
& H^{2}\left(\widetilde{S \times S} \times \mathbb{P}\left(H^{0}(E)\right), \bigwedge^{2} \tilde{E}_{2}^{*} \otimes \mathcal{O}(k-2)\right) \\
& =\left(p r_{1}^{*} H^{2}(S,-L) \oplus p r_{2}^{*} H^{2}(S,-L)\right) \otimes S^{k-2} H^{0}(S, E)^{*}, \\
& H^{2}\left(\widetilde{S \times S} \times \mathbb{P}\left(H^{0}(E)\right), \tilde{E}_{2}^{*} \otimes \mathcal{O}(k-1)\right) \\
& =\left(p r_{1}^{*} H^{2}\left(S, E^{*}\right) \oplus p r_{2}^{*} H^{2}\left(S, E^{*}\right)\right) \otimes S^{k-1} H^{0}(S, E)^{*} .
\end{aligned}
$$

Dualizing, we get

$$
\begin{aligned}
H^{2}\left(\widetilde{S \times S} \times \mathbb{P}\left(H^{0}(E)\right), \bigwedge_{2}^{2} \tilde{E}_{2}^{*} \otimes \mathcal{O}(k-2)\right)^{*} & \\
= & \left(H^{0}(S, L) \oplus H^{0}(S, L)\right) \otimes S^{k-2} H^{0}(S, E), \\
H^{2}\left(\widetilde{S \times S} \times \mathbb{P}\left(H^{0}(E)\right), \tilde{E}_{2}^{*} \otimes\right. & \mathcal{O}(k-1))^{*} \\
& =\left(H^{0}(S, E) \oplus H^{0}(S, E)\right) \otimes S^{k-1} H^{0}(S, E) .
\end{aligned}
$$

It is then immediate to check that the transpose of the map $\operatorname{int}(\sigma)$ is the map $\wedge \sigma$, so that $b$ ) translates into the condition that

$$
\begin{aligned}
\wedge \sigma:\left(H^{0}(S, E) \oplus H^{0}(S, E)\right) & \otimes S^{k-1} H^{0}(S, E) \\
& \rightarrow\left(H^{0}(S, L) \oplus H^{0}(S, L)\right) \otimes S^{k-2} H^{0}(S, E)
\end{aligned}
$$

is surjective. 
Now retracing through the isomorphisms given by Proposition 6, one checks that the map $\wedge \sigma$ is up to sign equal to the direct sum of two copies of the composed map

$$
\begin{gathered}
\mu: H^{0}(S, E) \otimes S^{k-1} H^{0}(S, E) \rightarrow H^{0}(S, E) \otimes H^{0}(S, E) \otimes S^{k-2} H^{0}(S, E) \\
\stackrel{\operatorname{det} \otimes i d}{\longrightarrow} H^{0}(S, L) \otimes S^{k-2} H^{0}(S, E) .
\end{gathered}
$$

Similarly statement d) dualizes as follows: by Proposition 6, the space

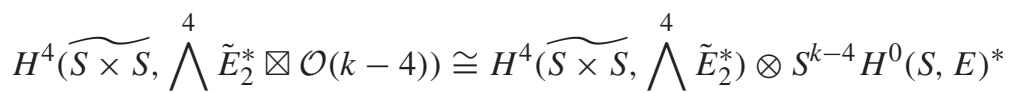

is dual to

$$
\operatorname{Ker}\left(H^{0}(S, L) \otimes H^{0}(S, L) \rightarrow H^{0}(S, 2 L)\right) \otimes S^{k-4} H^{0}(S, E) .
$$

Next, we know by Proposition 6, 4, that

$$
H^{4}\left(\widetilde{S \times S}, \bigwedge^{3} \tilde{E}_{2}^{*} \otimes \mathcal{O}(k-3)\right) \cong H^{4}\left(\widetilde{S \times S}, \bigwedge^{3} \tilde{E}_{2}^{*}\right) \otimes S^{k-3} H^{0}(S, E)^{*}
$$

admits a quotient which is dual to the direct sum of two copies of

$$
\left.\operatorname{Ker}\left(H^{0}(S, E) \otimes H^{0}(S, L) \rightarrow H^{0}(S, E \otimes L)\right) \otimes S^{k-3} H^{0}(S, E)\right) .
$$

Denoting by $Q_{E, L}:=\operatorname{Ker}\left(H^{0}(S, E) \otimes H^{0}(S, L) \rightarrow H^{0}(S, E \otimes L)\right), Q_{L, E}:=$ $\operatorname{Ker}\left(H^{0}(S, L) \otimes H^{0}(S, E) \rightarrow H^{0}(S, E \otimes L)\right)$ and $Q_{L, L}=\operatorname{Ker}\left(H^{0}(S, L) \otimes\right.$ $\left.H^{0}(S, L) \rightarrow H^{0}(S, 2 L)\right)$, we have an inclusion

$$
\left(Q_{L, E} \oplus Q_{E, L}\right) \otimes S^{k-3} H^{0}(S, E) \subset H^{4}\left(\widetilde{S \times S}, \bigwedge^{3} \tilde{E}_{2}^{*} \otimes \mathcal{O}(k-3)\right)^{*}
$$

and to prove d) it suffices to show that the map dual to $\operatorname{int}(\sigma)$ restricts on this subspace to a surjection

$$
\wedge \sigma:\left(Q_{L, E} \oplus Q_{E, L}\right) \otimes S^{k-3} H^{0}(S, E) \rightarrow Q_{L, L} \otimes S^{k-4} H^{0}(S, E) .
$$

But retracing through the isomorphisms of Proposition 6 and recalling the definition of $\sigma$, one checks easily that the first component

$$
\wedge \sigma_{1}: Q_{L, E} \otimes S^{k-3} H^{0}(S, E) \rightarrow Q_{L, L} \otimes S^{k-4} H^{0}(S, E)
$$

of the map above is the following composite

$$
\begin{aligned}
\mu^{\prime}: Q_{L, E} \otimes & S^{k-3} H^{0}(S, E) \subset H^{0}(S, L) \otimes H^{0}(S, E) \otimes S^{k-3} H^{0}(S, E) \rightarrow \\
& H^{0}(S, L) \otimes H^{0}(S, E) \otimes H^{0}(S, E) \otimes S^{k-4} H^{0}(S, E) \\
& \stackrel{i d \otimes \operatorname{det} \otimes i d}{\longrightarrow} H^{0}(S, L) \otimes H^{0}(S, L) \otimes S^{k-4} H^{0}(S, E),
\end{aligned}
$$

which takes obviously value in $Q_{L, L} \otimes S^{k-4} H^{0}(S, E)$, while the second component is equal to the first composed with the permutation exchanging factors on both sides. 
To conclude then that

$$
\wedge \sigma:\left(Q_{L, E} \oplus Q_{E, L}\right) \otimes S^{k-3} H^{0}(S, E) \rightarrow Q_{L, L} \otimes S^{k-4} H^{0}(S, E)
$$

is surjective, it suffices to show that

$$
\mu_{-}^{\prime}: Q_{L, E} \otimes S^{k-3} H^{0}(S, E) \rightarrow Q_{L, L}^{-} \otimes S^{k-4} H^{0}(S, E)
$$

and

$$
\mu_{+}^{\prime}: Q_{L, E} \otimes S^{k-3} H^{0}(S, E) \rightarrow Q_{L, L}^{+} \otimes S^{k-4} H^{0}(S, E)
$$

are surjective, where $Q_{L, L}^{+}$, (resp. $Q_{L, L}^{-}$) are the symmetric, resp. antisymmetric part of $Q_{L, L}$ and $\mu_{+}^{\prime}$ (resp. $\mu_{-}^{\prime}$ ) are the composition of $\mu^{\prime}$ with the projections on the symmetric (resp. antisymmetric) part of $Q_{L, L}$.

In conclusion, Theorem 2 (or the vanishing (4.21)) will be a consequence of the following propositions

Proposition 7. The composed map

$$
\begin{gathered}
\mu: H^{0}(S, E) \otimes S^{k-1} H^{0}(S, E) \rightarrow H^{0}(S, E) \otimes H^{0}(S, E) \otimes S^{k-2} H^{0}(S, E) \\
\stackrel{\text { det }}{\rightarrow} H^{0}(S, L) \otimes S^{k-2} H^{0}(S, E)
\end{gathered}
$$

is surjective.

Proposition 8. a) The map

$$
\mu_{-}^{\prime}: Q_{L, E} \otimes S^{k-3} H^{0}(S, E) \rightarrow Q_{L, L}^{-} \otimes S^{k-4} H^{0}(S, E)
$$

defined above is surjective.

b) The map

$$
\mu_{+}^{\prime}: Q_{L, E} \otimes S^{k-3} H^{0}(S, E) \rightarrow Q_{L, L}^{+} \otimes S^{k-4} H^{0}(S, E)
$$

defined above is surjective.

Proof of Proposition 7. Let $\alpha, \beta \in H^{0}(S, E)$ and $\gamma \in H^{0}(S, L)$ such that

$$
\gamma=\operatorname{det}(\alpha \wedge \beta) \text {. }
$$

Then we observe first that if $D \subset H^{0}(S, E)$ is the rank 2 vector subspace generated by $\alpha$ and $\beta$, we have

$$
\gamma \otimes S^{k-2} D \subset \operatorname{Im} \mu
$$

since the composite

$$
D \otimes S^{k-1} D \rightarrow D \otimes D \otimes S^{k-2} D \rightarrow \bigwedge^{2} D \otimes S^{k-2} D
$$

is surjective.

Recall now that the map det determines a morphism

$$
d: G_{2} \rightarrow \mathbb{P} H^{0}(S, L)
$$


which is surjective and finite since both spaces are of the same dimension $2 k$. The fiber $d^{-1}(\gamma)$ is then a finite subscheme $Z_{\gamma} \subset G_{2}$ which is the complete intersection of a space $W$ of hyperplane sections of the Grassmannian $G_{2}$.

Now by the above observation, and since $d$ is surjective, it suffices to show that the subspaces $S^{k-2} D$ for $D \in Z_{\gamma}$ generate $S^{k-2} H^{0}(S, E)$. If we dualize, this is equivalent to say that the dual map

$$
S^{k-2} H^{0}(S, E)^{*} \rightarrow \oplus_{D \in Z_{\gamma}} S^{k-2} D^{*}
$$

is injective. But this map identifies to the restriction

$$
H^{0}\left(G_{2}, S^{k-2} \mathcal{E}\right) \rightarrow H^{0}\left(Z_{\gamma}, S^{k-2} \mathcal{E}_{\mid Z_{\gamma}}\right),
$$

at least for a reduced $Z_{\gamma}$, which will be the case for a generic $\gamma$.

Hence it suffices to show that

$$
H^{0}\left(G_{2}, S^{k-2} \mathcal{E} \otimes \mathcal{I}_{Z_{\gamma}}\right)=0 .
$$

Now we use the Koszul resolution

$$
0 \rightarrow \bigwedge^{2 k} W \otimes \mathcal{L}^{-2 k} \rightarrow \ldots \rightarrow W \otimes \mathcal{L}^{-1} \rightarrow \mathcal{I}_{Z_{\gamma}} \rightarrow 0 .
$$

The vanishing (4.28) will then follow from the vanishing

$$
H^{i}\left(G_{2}, S^{k-2} \mathcal{E} \otimes \mathcal{L}^{-i-1}\right), i=0,2 k-1
$$

which is proved in Proposition 9 of the appendix.

Proof of Proposition 8, a). Notice first that the natural composed map

$$
\begin{gathered}
\bigwedge^{3} H^{0}(S, E) \rightarrow \bigwedge^{2} H^{0}(S, E) \otimes H^{0}(S, E) \\
\stackrel{\operatorname{det} \otimes i d}{\longrightarrow} H^{0}(S, L) \otimes H^{0}(S, E)
\end{gathered}
$$

has its image contained in $Q_{L, E}$. Hence it suffices to show that the following composite

$$
\begin{aligned}
& \mu^{\prime \prime}: \bigwedge^{3} H^{0}(S, E) \otimes S^{k-3} H^{0}(S, E) \rightarrow \bigwedge^{2} H^{0}(S, E) \otimes H^{0}(S, E) \otimes S^{k-3} H^{0}(S, E) \\
& \stackrel{\operatorname{det} \otimes \mu}{\longrightarrow} H^{0}(S, L) \otimes H^{0}(S, L) \otimes S^{k-4} H^{0}(S, E) \rightarrow \bigwedge^{0}(S, L) \otimes S^{k-4} H^{0}(S, E)
\end{aligned}
$$

is surjective.

Now note that for $\alpha_{1}, \alpha_{2}, \alpha_{3} \in H^{0}(S, E)$

$\mu^{\prime \prime}\left(\alpha_{1} \wedge \alpha_{2} \wedge \alpha_{3} \otimes \alpha_{3}^{k-3}\right)=2(k-3) \operatorname{det}\left(\alpha_{2} \wedge \alpha_{3}\right) \wedge \operatorname{det}\left(\alpha_{1} \wedge \alpha_{3}\right) \otimes \alpha_{3}^{k-4}$. 
Fix now $\gamma \in H^{0}(S, L)$ and consider the set of couples $\left(\alpha_{1}, \alpha_{3}\right)$ such that

$$
\operatorname{det}\left(\alpha_{1} \wedge \alpha_{3}\right)=\gamma
$$

For any $\alpha_{2}$ and any such $\left(\alpha_{1}, \alpha_{3}\right)$, we have

$$
\mu^{\prime \prime}\left(\alpha_{1} \wedge \alpha_{2} \wedge \alpha_{3} \otimes \alpha_{3}^{k-3}\right)=2(k-3) \operatorname{det}\left(\alpha_{2} \wedge \alpha_{3}\right) \wedge \gamma \otimes \alpha_{3}^{k-4} .
$$

Note that the vector $\alpha_{3}$ for such pairs takes arbitrary value in some of the lines $D \in Z_{\gamma}$, where the notations are as in the previous proposition.

Now we have the map

$$
\mu^{\prime \prime \prime}: H^{0}(S, E) \otimes S^{k-3} H^{0}(S, E) \rightarrow H^{0}(S, L) \otimes S^{k-4} H^{0}(S, E)
$$

analogous to $\mu$ and the formula above shows that

$$
\mu^{\prime \prime}\left(\alpha_{1} \wedge \alpha_{2} \wedge \alpha_{3} \otimes \alpha_{3}^{k-3}\right)=2 \gamma \wedge \mu^{\prime \prime \prime}\left(\alpha_{2} \otimes \alpha_{3}^{k-3}\right)
$$

With the same proof as in the previous proposition, one shows now that the $S^{k-3} D, D \in Z_{\gamma}$ generate $S^{k-3} H^{0}(S, E)$ and that $\mu^{\prime \prime \prime}$ is surjective. Hence the $\alpha_{2} \otimes \alpha_{3}^{k-3}, \alpha_{3} \in D, D \in Z_{\gamma}$ generate $H^{0}(S, E) \otimes S^{k-3} H^{0}(S, E)$ and the $\mu^{\prime \prime \prime}\left(\alpha_{2} \otimes\right.$ $\left.\alpha_{3}^{k-3}\right), \alpha_{3} \in D, D \in Z_{\gamma}$ generate by the surjectivity of $\mu^{\prime \prime \prime}$ the space $H^{0}(S, L) \otimes$ $S^{k-4} H^{0}(S, E)$. Hence $\operatorname{Im} \mu^{\prime \prime}$ contains $\gamma \wedge H^{0}(S, L) \otimes S^{k-4} H^{0}(S, E)$, and since $\gamma$ was generic, we conclude that $\mu^{\prime \prime}$ is surjective.

Proof of Proposition 8, $b$ ). We want to prove that

$$
\mu_{+}^{\prime}: Q_{L, E} \otimes S^{k-3} H^{0}(S, E) \rightarrow Q_{L, L}^{+} \otimes S^{k-4} H^{0}(S, E)
$$

is surjective. Denote similarly, for $C$ a generic member of $|L|$,

$$
\begin{gathered}
Q_{K_{C}, E}:=\operatorname{Ker}\left(H^{0}\left(C, K_{C}\right) \otimes H^{0}\left(C, E_{\mid C}\right) \rightarrow H^{0}\left(C, E \otimes K_{C}\right)\right), \\
Q_{K_{C}, K_{C}}^{+}:=\operatorname{Ker}\left(S^{2} H^{0}\left(C, K_{C}\right) \rightarrow H^{0}\left(C, K_{C}^{\otimes 2}\right)\right) .
\end{gathered}
$$

Then we can define

$$
\mu_{+, C}^{\prime}: Q_{K_{C}, E} \otimes S^{k-3} H^{0}\left(C, E_{\mid C}\right) \rightarrow Q_{K_{C}, K_{C}}^{+} \otimes S^{k-4} H^{0}\left(C, E_{\mid C}\right)
$$

as the composite

$$
\begin{aligned}
Q_{K_{C}, E} \otimes & S^{k-3} H^{0}\left(C, E_{\mid C}\right) \subset H^{0}\left(C, K_{C}\right) \otimes H^{0}\left(C, E_{\mid C}\right) \otimes S^{k-3} H^{0}\left(C, E_{\mid C}\right) \\
& \rightarrow H^{0}\left(C, K_{C}\right) \otimes H^{0}\left(C, E_{\mid C}\right) \otimes H^{0}\left(C, E_{\mid C}\right) \otimes S^{k-4} H^{0}\left(C, E_{\mid C}\right) \\
& \stackrel{i d \otimes \operatorname{det} \otimes i d}{\longrightarrow} H^{0}\left(C, K_{C}\right) \otimes H^{0}\left(C, K_{C}\right) \otimes S^{k-4} H^{0}\left(C, E_{\mid C}\right) \\
& \rightarrow S^{2} H^{0}\left(C, K_{C}\right) \otimes S^{k-4} H^{0}\left(C, E_{\mid C}\right) .
\end{aligned}
$$

Now the restriction map $H^{0}(S, E) \rightarrow H^{0}\left(C, E_{\mid C}\right)$ is an isomorphism, and the restriction map $H^{0}(S, L) \rightarrow H^{0}\left(C, K_{C}\right)$ is surjective with kernel $\sigma$, the defining equation of $C$. Hence the restrictions induce a surjection

$$
Q_{L, E} \rightarrow Q_{K_{C}, E}
$$


and an isomorphism

$$
Q_{L, L}^{+} \cong Q_{K_{C}, K_{C}}^{+},
$$

and it suffices to show that $\mu_{+, C}^{\prime}$ is surjective. A fortiori it suffices to show that the composite

$$
\begin{gathered}
\mu_{C}^{\prime}: Q_{K_{C}, E} \otimes S^{k-3} H^{0}\left(C, E_{\mid C}\right) \subset H^{0}\left(C, K_{C}\right) \otimes H^{0}\left(C, E_{\mid C}\right) \otimes S^{k-3} H^{0}\left(C, E_{\mid C}\right) \\
\rightarrow H^{0}\left(C, K_{C}\right) \otimes H^{0}\left(C, E_{\mid C}\right) \otimes H^{0}\left(C, E_{\mid C}\right) \otimes S^{k-4} H^{0}\left(C, E_{\mid C}\right) \\
\stackrel{i d \otimes \operatorname{det} \otimes i d}{\longrightarrow} H^{0}\left(C, K_{C}\right) \otimes H^{0}\left(C, K_{C}\right) \otimes S^{k-4} H^{0}\left(C, E_{\mid C}\right)
\end{gathered}
$$

which takes value in $Q_{K_{C}, K_{C}}:=\operatorname{Ker}\left(H^{0}\left(C, K_{C}\right)^{\otimes 2} \rightarrow H^{0}\left(C, K_{C}^{\otimes 2}\right)\right)$, is surjective on this last space.

Let us now consider the following diagram of exact sequences

$$
\begin{gathered}
0 \rightarrow Q_{K_{C}, E} \otimes S^{k-3} H^{0}\left(C, E_{\mid C}\right) \rightarrow H^{0}\left(C, K_{C}\right) \otimes H^{0}\left(C, E_{\mid C}\right) \otimes S^{k-3} H^{0}\left(C, E_{\mid C}\right) \\
\mu_{C}^{\prime} \downarrow \\
0 \rightarrow Q_{K_{C}, K_{C}} \otimes S^{k-4} H^{0}\left(C, E_{\mid C}\right) \rightarrow H_{C} \downarrow \\
\rightarrow H^{0}\left(C, E \otimes K_{C}\right) \otimes H^{0}\left(C, K_{C}\right) \otimes S^{k-4} H^{0}\left(C, E_{\mid C}\right) \\
\mu_{C, K_{C} \downarrow} \downarrow-3 H^{0}\left(C, E_{\mid C}\right) \rightarrow 0 \\
\rightarrow H^{0}\left(C, K_{C}^{\otimes 2}\right) \otimes S^{k-4} H^{0}\left(C, E_{\mid C}\right) \rightarrow 0
\end{gathered}
$$

where the vertical maps $\mu_{C}$ and $\mu_{C, K_{C}}$ are defined in a way similar to $\mu$ e.g $\mu_{C}$ is the composite

$H^{0}\left(C, E_{\mid C}\right) \otimes S^{k-3} H^{0}\left(C, E_{\mid C}\right) \subset H^{0}\left(C, E_{\mid C}\right) \otimes H^{0}\left(C, E_{\mid C}\right) \otimes S^{k-4} H^{0}\left(C, E_{\mid C}\right)$

$$
\stackrel{\operatorname{det} \otimes i d}{\longrightarrow} H^{0}\left(C, K_{C}\right) \otimes S^{k-4} H^{0}\left(C, E_{\mid C}\right),
$$

and $\mu_{C, K_{C}}$ is defined similarly with a twist by $K_{C}$.

One checks easily the surjectivity of the multiplication maps on the left.

The proof of Proposition 7 shows as well that $\mu_{C}$ is surjective, as is $\mu_{C, K_{C}}$ by the commutativity of the diagram above. Hence the surjectivity of $\mu_{C}^{\prime}$ will follow by diagram chasing from the surjectivity of the induced multiplication map

$$
H^{0}\left(C, K_{C}\right) \otimes \operatorname{Ker} \mu_{C} \rightarrow \operatorname{Ker} \mu_{C, K_{C}} .
$$

In what follows we will use again the notation $H^{0}(E)$ for $H^{0}(S, E)=H^{0}\left(C, E_{\mid C}\right)$. Define the vector bundle $\mathcal{Q}$ on $C$ as the kernel of the surjective composite morphism of vector bundles

$$
S^{k-3} H^{0}(E) \otimes E \subset S^{k-4} H^{0}(E) \otimes H^{0}(E) \otimes E \stackrel{i d \otimes d e t}{\longrightarrow} S^{k-4} H^{0}(E) \otimes K_{C} .
$$

Then we clearly have

$$
\operatorname{Ker} \mu_{C}=H^{0}(C, \mathcal{Q}), \operatorname{Ker} \mu_{C, K_{C}}=H^{0}\left(C, \mathcal{Q} \otimes K_{C}\right)
$$


so that the surjectivity of the map (4.30) is equivalent to the surjectivity of the multiplication map

$$
H^{0}(C, \mathcal{Q}) \otimes H^{0}\left(C, K_{C}\right) \rightarrow H^{0}\left(C, \mathcal{Q} \otimes K_{C}\right) .
$$

Now we proceed as follows: let $\sigma \in H^{0}(S, L)$ be the defining equation for $C$. Recall the finite reduced subscheme $Z_{\sigma}=d^{-1}(\sigma) \subset G_{2}$ made of the rank 2 vector subspaces $D$ of $H^{0}(S, E)$ such that $\operatorname{det} D=\sigma$. For each such $D$, there is a line subbundle $L_{D}$ of $E$ on $C$, of degree $k+1$ with two sections without common zeroes (see Sect. 2). The space $D$ identifies naturally to $H^{0}\left(C, L_{D}\right)$.

Clearly the image of the inclusion

$$
S^{k-3} H^{0}\left(C, L_{D}\right) \otimes L_{D} \subset S^{k-3} H^{0}(E) \otimes E
$$

is contained in $\mathcal{Q}$.

Let now

$$
\mathcal{N}:=\oplus_{D \in Z_{\sigma}} S^{k-3} D \otimes L_{D} .
$$

Then by the observation above we have a morphism

$$
\alpha: \mathcal{N} \rightarrow \mathcal{Q}
$$

The surjectivity of 4.31 will follow from the following three lemmas:

Lemma 4. The morphism $\alpha$ is surjective.

Denoting $\mathcal{M}:=\operatorname{Ker} \alpha$ we also prove

Lemma 5. The vector bundle $\mathcal{M}$ is generated by its sections.

Lemma 6. The space $H^{0}(C, \mathcal{M})$ is generated by the subspaces $H^{0}(C, \mathcal{M}(-x))$, $x \in C$.

We explain first how these three lemmas imply our result. Using the exact sequence

$$
0 \rightarrow \mathcal{M} \rightarrow \mathcal{N} \rightarrow \mathcal{Q} \rightarrow 0
$$

given by Lemma 4, we see that the map (4.31) will be surjective if the multiplication map

$$
H^{0}(C, \mathcal{N}) \otimes H^{0}\left(C, K_{C}\right) \rightarrow H^{0}\left(C, \mathcal{N} \otimes K_{C}\right)
$$

is surjective, and $H^{1}\left(C, \mathcal{M} \otimes K_{C}\right)=0$.

The first condition is easy to check. Indeed $\mathcal{N}$ is a direct sum of line bundles $L_{D}$ corresponding to $g_{k+1}^{1}$ 's on $C$, and the result is easy to prove for each of them. As for the second condition, it is equivalent to $H^{0}\left(C, \mathcal{M}^{*}\right)=0$ by Serre's duality. But since $\mathcal{M}$ is generated by sections by Lemma 5 , we have an inclusion

$$
H^{0}\left(C, \mathcal{M}^{*}\right) \subset H^{0}(C, \mathcal{M})^{*} .
$$

The image of this inclusion obviously vanishes on each subspace $H^{0}(C, \mathcal{M}(-x))$, hence it must be 0 since we know by Lemma 6 that these subspaces generate $H^{0}(C, \mathcal{M})$. 
To conclude the proof of $8, \mathrm{~b}$ ) it remains only to prove these three lemmas.

Proof of Lemma 4. First of all we note that the bundle $\mathcal{Q}$ is generated by its sections, since there is a natural surjection

$$
S^{k-2} H^{0}(E) \otimes \mathcal{O}_{C} \rightarrow \mathcal{Q} \rightarrow 0 .
$$

Hence it suffices to show that the map

$$
H^{0}(C, \mathcal{N}) \rightarrow H^{0}(C, \mathcal{Q})
$$

is surjective.

But by definition

$$
H^{0}(C, \mathcal{Q})=\operatorname{Ker}\left(H^{0}(E) \otimes S^{k-3} H^{0}(E) \stackrel{\mu_{C}}{\rightarrow} H^{0}\left(C, K_{C}\right) \otimes S^{k-4} H^{0}(E)\right)
$$

and

$$
H^{0}(C, \mathcal{N})=\oplus_{D \in Z_{\sigma}} D \otimes S^{k-3} D .
$$

Hence we need to show that the sequence

$$
\oplus_{D \in Z_{\sigma}} D \otimes S^{k-3} D \rightarrow H^{0}(E) \otimes S^{k-3} H^{0}(E) \stackrel{\mu_{C}}{\rightarrow} H^{0}\left(C, K_{C}\right) \otimes S^{k-4} H^{0}(E)
$$

is exact at the middle. Again this will follow from a cohomological computation on the Grassmannian $G_{2}$. Indeed, the notations being as in the proof of Propositions 4 and 7 , the sequence above dualizes as

$$
\begin{array}{r}
I_{Z_{\sigma}}(\mathcal{L}) \otimes S^{k-4} H^{0}\left(G_{2}, \mathcal{E}\right) \rightarrow H^{0}\left(G_{2}, \mathcal{E}\right) \otimes S^{k-3} H^{0}\left(G_{2}, \mathcal{E}\right) \\
\rightarrow H^{0}\left(\mathcal{E} \otimes S^{k-3} \mathcal{E}_{\mid Z_{\sigma}}\right)
\end{array}
$$

where the map

$$
I_{Z_{\sigma}}(\mathcal{L}) \otimes S^{k-4} H^{0}\left(G_{2}, \mathcal{E}\right) \rightarrow H^{0}\left(G_{2}, \mathcal{E}\right) \otimes S^{k-3} H^{0}\left(G_{2}, \mathcal{E}\right)
$$

is composed of the inclusion

$$
\begin{gathered}
I_{Z_{\sigma}}(\mathcal{L}) \otimes S^{k-4} H^{0}\left(G_{2}, \mathcal{E}\right) \subset H^{0}\left(G_{2}, \mathcal{L}\right) \otimes S^{k-4} H^{0}\left(G_{2}, \mathcal{E}\right) \\
\cong \wedge^{2} H^{0}\left(G_{2}, \mathcal{E}\right) \otimes S^{k-4} H^{0}\left(G_{2}, \mathcal{E}\right)
\end{gathered}
$$

and of the (Koszul) map

$$
\wedge^{2} H^{0}\left(G_{2}, \mathcal{E}\right) \otimes S^{k-4} H^{0}\left(G_{2}, \mathcal{E}\right) \rightarrow H^{0}\left(G_{2}, \mathcal{E}\right) \otimes S^{k-3} H^{0}\left(G_{2}, \mathcal{E}\right) .
$$

One checks easily that $H^{0}\left(G_{2}, \mathcal{E}\right) \otimes S^{k-3} H^{0}\left(G_{2}, \mathcal{E}\right) \cong H^{0}\left(G_{2}, \mathcal{E} \otimes S^{k-3} \mathcal{E}\right)$. Hence the kernel in the middle identifies to $H^{0}\left(G_{2}, \mathcal{E} \otimes S^{k-3} \mathcal{E} \otimes \mathcal{I}_{Z_{\sigma}}\right)$. Furthermore $S^{k-4} H^{0}\left(G_{2}, \mathcal{E}\right) \cong H^{0}\left(G_{2}, S^{k-4} \mathcal{E}\right)$ identifies to $H^{0}\left(G_{2}, \mathcal{E} \otimes S^{k-3} \mathcal{E} \otimes \mathcal{L}^{-1}\right)$ via the (Koszul) inclusion

$$
S^{k-4} \mathcal{E} \otimes \mathcal{L}=S^{k-4} \mathcal{E} \otimes \bigwedge^{2} \mathcal{E} \subset \mathcal{E} \otimes S^{k-3} \mathcal{E}
$$


Hence the exactness at the middle of the sequence 4.32 will follow from the surjectivity of the multiplication map

$$
H^{0}\left(G_{2}, \mathcal{E} \otimes S^{k-3} \mathcal{E} \otimes \mathcal{L}^{-1}\right) \otimes I_{Z_{\sigma}}(\mathcal{L}) \rightarrow H^{0}\left(G_{2}, \mathcal{E} \otimes S^{k-3} \mathcal{E} \otimes \mathcal{I}_{Z_{\sigma}}\right) .
$$

Now let $W:=I_{Z_{\sigma}}(\mathcal{L})$. The Koszul resolution of $\mathcal{I}_{Z_{\sigma}}$

$$
0 \rightarrow \bigwedge^{2 k} W \otimes \mathcal{L}^{-2 k} \rightarrow \ldots \rightarrow W \otimes \mathcal{L}^{-1} \rightarrow \mathcal{I}_{Z_{\sigma}} \rightarrow 0
$$

twisted by $\mathcal{E} \otimes S^{k-3} \mathcal{E}$ shows that the surjectivity of (4.33) will hold if we know that

$$
H^{i}\left(G_{2}, \mathcal{E} \otimes S^{k-3} \mathcal{E} \otimes \mathcal{L}^{-i-1}\right)=0,1 \leq i<2 k .
$$

Since we have the exact sequence

$$
0 \rightarrow S^{k-4} \mathcal{E} \otimes \mathcal{L} \rightarrow \mathcal{E} \otimes S^{k-3} \mathcal{E} \rightarrow S^{k-2} \mathcal{E} \rightarrow 0,
$$

it suffices to know that

$$
H^{i}\left(G_{2}, S^{k-4} \mathcal{E} \otimes \mathcal{L}^{-i}\right)=0,1 \leq i<2 k,
$$

and

$$
H^{i}\left(G_{2}, S^{k-2} \mathcal{E} \otimes \mathcal{L}^{-i-1}\right)=0,1 \leq i<2 k .
$$

This is proved in Proposition 9.

Proof of Lemma 5. The bundles $\mathcal{N}$ and $\mathcal{Q}$ are generated by global sections. To prove that $\mathcal{M}$ is generated by global sections, it suffices to prove that for any $x \in C$, the restriction map $H^{0}(C, \mathcal{N}(-x)) \rightarrow H^{0}(C, \mathcal{Q}(-x))$ is surjective. For each $g_{k+1}^{1} L_{D}$ on $C$, denote by $\sigma_{D, x} \in H^{0}\left(C, L_{D}\right) \cong D$ a generator for $H^{0}\left(C, L_{D}(-x)\right)$. We need to show the exactness of the sequence

$$
\begin{aligned}
\oplus_{D \in Z_{\sigma}} \sigma_{D, x} \otimes S^{k-3} D & \rightarrow H^{0}(C, E(-x)) \otimes S^{k-3} H^{0}(E) \\
& \stackrel{\mu_{C}}{\rightarrow} H^{0}\left(C, K_{C}(-x)\right) \otimes S^{k-4} H^{0}(E) .
\end{aligned}
$$

(Indeed, by definition, $\oplus_{D \in Z_{\sigma}} \sigma_{D, x} \otimes S^{k-3} D$ identifies to $H^{0}(C, \mathcal{N}(-x))$ and

$$
\operatorname{Ker}\left(H^{0}(C, E(-x)) \otimes S^{k-3} H^{0}(E) \stackrel{\mu_{C}}{\rightarrow} H^{0}\left(C, K_{C}(-x)\right) \otimes S^{k-4} H^{0}(E)\right)
$$

identifies to $H^{0}(C, \mathcal{Q}(-x))$.)

Denote by $K_{x} \subset H^{0}(E)$ the subspace $H^{0}(C, E(-x))$. Note that via the identification $H^{0}\left(C, L_{D}\right)=D \subset H^{0}(E), \sigma_{D, x}$ becomes a generator of the 1-dimensional vector space $D \cap K_{x}$. Furthermore, $K_{x}$ being of codimension 2 in $H^{0}(E)$ determines a section $\tau_{x} \in \bigwedge^{2} H^{0}(E)^{*}$ up to a coefficient. Clearly $\tau_{x}$ belongs to $H^{0}\left(C, K_{C}\right)^{*} \subset \bigwedge^{2} H^{0}(E)^{*}$ and identifies also to the linear form on $H^{0}\left(C, K_{C}\right)$ defining $H^{0}\left(C, K_{C}(-x)\right)$. Let $G_{x} \subset G_{2}$ be the hyperplane section defined by $\tau_{x}$. The scheme $Z_{\sigma}$ is a complete intersection of hyperplane sections of $G_{x}$. The variety $G_{x}$ admits a desingularization $P_{x} \stackrel{g}{\rightarrow} G_{x}$ defined as

$$
P_{x}=\left\{(u, \Delta) \in \mathbb{P}\left(K_{x}\right) \times G_{2}, u \in \Delta \cap K_{x}\right\} .
$$


Note that if<smiles>CCCCCCPCC</smiles>

is the incidence variety, $P_{x}$ can also be defined as $f^{-1}\left(\mathbb{P}\left(K_{x}\right)\right) \subset P$.

Each line $D$ parametrized by $Z_{\sigma}$ meets $K_{x}$ along a one dimensional vector space, because $L_{D}$ has no base-point, so that $H^{0}\left(L_{D}(-x)\right) \cong D \cap K_{x}$ is 1dimensional. It follows that the scheme $Z_{\sigma}$ can also be seen as the complete intersection in $P_{x}$ of hypersurfaces in $\left|g^{*} \mathcal{L}\right|$.

We now dualize the sequence (4.34). The space $H^{0}\left(C, K_{C}(-x)\right)$ admits for dual the space $W \subset H^{0}\left(P_{x}, g^{*} \mathcal{L}\right)$ defining $Z_{\sigma} \subset P_{x}$. The vector space $<\sigma_{D, x}>^{*}$ identifies clearly to the fiber of the line bundle $H_{x}:=f^{*} \mathcal{O}_{\mathbb{P}\left(K_{x}\right)}(1)$ at the point $D \in Z_{\sigma}$. Hence our sequence dualizes as

$$
\begin{array}{r}
W \otimes S^{k-4} H^{0}(E)^{*} \rightarrow f^{*} H^{0}\left(\mathbb{P}\left(K_{x}\right),\right. \\
\rightarrow(1)) \otimes H^{0}\left(P_{x}, g^{*} S^{k-3} \mathcal{E}\right) \\
\rightarrow H^{0}\left(S^{k-3} \mathcal{E} \otimes H_{x} \mid Z_{\sigma}\right)
\end{array}
$$

The second space in this sequence is easily shown to identify to $H^{0}\left(P_{x}, g^{*} S^{k-3} \mathcal{E} \otimes\right.$ $H_{x}$ ), so that the kernel at the middle is equal to

$$
H^{0}\left(P_{x}, g^{*} S^{k-3} \mathcal{E} \otimes H_{x} \otimes \mathcal{I}_{Z_{\sigma}}\right) .
$$

The first map in (4.35) is induced by the isomorphism

$$
S^{k-4} H^{0}(E)^{*} \cong H^{0}\left(P_{x}, g^{*} S^{k-4} \mathcal{E}\right),
$$

the multiplication

$$
W \otimes H^{0}\left(P_{x}, g^{*} S^{k-4} \mathcal{E}\right) \rightarrow H^{0}\left(P_{x}, g^{*}\left(S^{k-4} \mathcal{E} \otimes \mathcal{L}\right) \otimes \mathcal{I}_{Z_{\sigma}}\right)
$$

and the composed bundle map

$$
g^{*}\left(S^{k-4} \mathcal{E} \otimes \mathcal{L}\right) \rightarrow g^{*}\left(S^{k-3} \mathcal{E} \otimes \mathcal{E}\right) \rightarrow g^{*} S^{k-3} \mathcal{E} \otimes H_{x},
$$

where the last map is induced by the natural surjective map $g^{*} \mathcal{E} \rightarrow H_{x}$.

The exactness of (4.35) will then follow from the surjectivity of

$$
W \otimes H^{0}\left(P_{x}, g^{*}\left(S^{k-3} \mathcal{E} \otimes \mathcal{L}^{-1}\right) \otimes H_{x}\right) \rightarrow H^{0}\left(P_{x}, g^{*} S^{k-3} \mathcal{E} \otimes H_{x} \otimes \mathcal{I}_{Z_{\sigma}}\right)
$$

and from the equality

$$
H^{0}\left(P_{x}, g^{*}\left(S^{k-3} \mathcal{E} \otimes \mathcal{L}^{-1}\right) \otimes H_{x}\right)=H^{0}\left(P_{x}, g^{*} S^{k-4} \mathcal{E}\right) .
$$

This last equality is proved as follows: on $P_{x}$ we have the exact sequence

$$
0 \rightarrow g^{*} \mathcal{L} \otimes H_{x}^{-1} \rightarrow g^{*} \mathcal{E} \rightarrow H_{x} \rightarrow 0
$$


which gives

$$
0 \rightarrow g^{*} S^{k-4} \mathcal{E} \otimes g^{*} \mathcal{L} \otimes H_{x}^{-1} \rightarrow g^{*} S^{k-3} \mathcal{E} \rightarrow H_{x}^{k-3} \rightarrow 0 .
$$

Tensoring this with $H_{x} \otimes \mathcal{L}^{-1}$ we get

$$
0 \rightarrow g^{*} S^{k-4} \mathcal{E} \rightarrow g^{*}\left(S^{k-3} \mathcal{E} \otimes \mathcal{L}^{-1}\right) \otimes H_{x} \rightarrow g^{*} \mathcal{L}^{-1} \otimes H_{x}^{k-2} \rightarrow 0 .
$$

But the right hand side has no non-zero sections since it is of negative degree on the fibers of $f$. Hence the equality (4.37).

Since $Z_{\sigma} \subset P_{x}$ is the complete intersection of the space $W$ of sections of $g^{*} \mathcal{L}$, we have a Koszul resolution of $\mathcal{I}_{Z_{\sigma}}$, which takes the form

$$
0 \rightarrow \bigwedge^{2 k-1} W \otimes g^{*} \mathcal{L}^{-2 k-1} \rightarrow \ldots \rightarrow W \otimes g^{*} \mathcal{L}^{-1} \rightarrow \mathcal{I}_{Z_{\sigma}} \rightarrow 0 .
$$

We can tensor it with $g^{*} S^{k-3} \mathcal{E} \otimes H_{x}$, and the surjectivity of the map (4.36) will follow from the following vanishing

$$
H^{i}\left(P_{x}, g^{*}\left(S^{k-3} \mathcal{E} \otimes \mathcal{L}^{-i-1}\right) \otimes H_{x}\right)=0,1 \leq i<2 k-1=\operatorname{dim} P_{x} .
$$

Recall now that $P_{x} \subset P$ is the complete intersection of two sections of $H=$ $f^{*} \mathcal{O}_{\mathbb{P}\left(H^{0}(E)\right)}(1)$, with $H_{x}=H_{\mid P_{x}}$. The vanishing (4.38) will then follow from

$$
\begin{gathered}
H^{i}\left(P, g^{*}\left(S^{k-3} \mathcal{E} \otimes \mathcal{L}^{-i-1}\right) \otimes H\right)=0,1 \leq i<2 k-1 \\
H^{i+1}\left(P, g^{*}\left(S^{k-3} \mathcal{E} \otimes \mathcal{L}^{-i-1}\right)\right)=0,1 \leq i<2 k-1 \\
H^{i+2}\left(P, g^{*}\left(S^{k-3} \mathcal{E} \otimes \mathcal{L}^{-i-1}\right) \otimes H^{-1}\right)=0,1 \leq i<2 k-1 .
\end{gathered}
$$

The second equality follows immediately from the Proposition 9, and the third is obvious since $H^{-1}$ has no cohomology on the fibers of $g$. The first equality is proven as follows: we have

$$
\left.H^{i}\left(P, g^{*} S^{k-3} \mathcal{E} \otimes \mathcal{L}^{-i-1}\right) \otimes H\right)=H^{i}\left(G_{2}, S^{k-3} \mathcal{E} \otimes \mathcal{E} \otimes \mathcal{L}^{-i-1}\right),
$$

since $R^{0} g_{*} H=\mathcal{E}$. Now we have the exact sequence on $G_{2}$

$$
0 \rightarrow S^{k-4} \mathcal{E} \otimes \mathcal{L} \rightarrow S^{k-3} \mathcal{E} \otimes \mathcal{E} \rightarrow S^{k-2} \mathcal{E} \rightarrow 0 .
$$

Hence the needed equality will follow from the vanishings

$$
\begin{gathered}
H^{i}\left(G_{2}, S^{k-4} \mathcal{E} \otimes \mathcal{L}^{-i}\right)=0, \\
H^{i}\left(G_{2}, S^{k-2} \mathcal{E} \otimes \mathcal{L}^{-i-1}\right)=0,
\end{gathered}
$$

for $1 \leq i<2 k-1$, which are proved in proposition (9). Hence Lemma 5 is proven. 
Proof of Lemma 6 . Let $x_{1}, \ldots, x_{2 k-1}$ be points of $C$ in general position. We will show that the natural map

$$
\oplus_{i} H^{0}\left(C, \mathcal{M}\left(-x_{i}\right)\right) \rightarrow H^{0}(C, \mathcal{M})
$$

is surjective.

Recall that

$$
H^{0}(C, \mathcal{M})=\operatorname{Ker} \oplus_{D \in Z_{\sigma}} S^{k-3} D \otimes D \rightarrow S^{k-3} H^{0}(E) \otimes H^{0}(E) .
$$

It follows from this, using the identifications

$$
H^{0}(E)^{*}=H^{0}\left(G_{2}, \mathcal{E}\right), D^{*} \cong \mathcal{E}_{D}
$$

that

$$
\begin{gathered}
H^{0}(C, \mathcal{M})^{*}=\text { Coker } H^{0}\left(G_{2}, S^{k-3} \mathcal{E} \otimes \mathcal{E}\right) \rightarrow H^{0}\left(S^{k-3} \mathcal{E} \otimes \mathcal{E}_{\mid Z_{\sigma}}\right) \\
=H^{1}\left(G_{2}, S^{k-3} \mathcal{E} \otimes \mathcal{E} \otimes \mathcal{I}_{Z_{\sigma}}\right) .
\end{gathered}
$$

Similarly

$$
H^{0}\left(C, \mathcal{M}\left(-x_{i}\right)\right)=\operatorname{Ker} \oplus_{D \in Z_{\sigma}} S^{k-3} D \otimes \sigma_{D, x_{i}} \rightarrow S^{k-3} H^{0}(E) \otimes K_{x_{i}}
$$

which, with the notations of the previous proof, dualizes to

$$
\begin{gathered}
H^{0}\left(C, \mathcal{M}\left(-x_{i}\right)\right)^{*}=\operatorname{Coker}\left(H^{0}\left(P_{x_{i}}, g^{*} S^{k-3} \mathcal{E} \otimes H_{x_{i}}\right) \rightarrow H^{0}\left(Z_{\sigma}, S^{k-3} \mathcal{E} \otimes H_{x_{i}}\right)\right) \\
=H^{1}\left(P_{x_{i}}, g^{*} S^{k-3} \mathcal{E} \otimes H_{x_{i}} \otimes \mathcal{I}_{Z_{\sigma}}\right),
\end{gathered}
$$

where we view $Z_{\sigma}$ as a subscheme of $P_{x_{i}}$ as well. Hence we have to show that the natural map (induced by the morphism $g^{*} \mathcal{E} \rightarrow H_{x_{i}}$ on $P_{x_{i}}$ )

$$
H^{1}\left(G_{2}, S^{k-3} \mathcal{E} \otimes \mathcal{E} \otimes \mathcal{I}_{Z_{\sigma}}\right) \rightarrow \oplus_{i} H^{1}\left(P_{x_{i}}, g^{*} S^{k-3} \mathcal{E} \otimes H_{x_{i}} \otimes \mathcal{I}_{Z_{\sigma}}\right)
$$

is injective.

Let $R \subset G_{2}$ be the curve which is the complete intersection of the sections $\sigma_{x_{i}} \in H^{0}\left(G_{2}, \mathcal{L}\right)$. We have first

Fact. The restriction map

$$
H^{0}\left(G_{2}, S^{k-3} \mathcal{E} \otimes \mathcal{E}\right) \rightarrow H^{0}\left(R, S^{k-3} \mathcal{E} \otimes \mathcal{E}_{\mid R}\right)
$$

is surjective.

Using the Koszul resolution of $\mathcal{I}_{D}$ this is obtained by application of the Proposition 9. 
From this we conclude that the restriction map

$$
H^{1}\left(G_{2}, S^{k-3} \mathcal{E} \otimes \mathcal{E} \otimes \mathcal{I}_{Z_{\sigma}}\right) \rightarrow H^{1}\left(R, S^{k-3} \mathcal{E} \otimes \mathcal{E}_{\mid R} \otimes \mathcal{I}_{Z_{\sigma}}\right)
$$

is injective.

Consider now the fibered product

$$
\tilde{R}:=P_{x_{1}} \times_{G_{2}} \times \ldots \times_{G_{2}} P_{x_{2 k-1}} .
$$

Denote by $\tilde{g}: \tilde{R} \rightarrow R \subset G_{2}$ the natural morphism. One shows easily that the curve $\tilde{R}$ is isomorphic to $R$ excepted over the intersection of $R$ with the Grassmannian of lines in $\mathbb{P}\left(K_{x_{i}}\right)$ for some $i$. Here $R$ has nodes, which are replaced in $\tilde{R}$ by lines.

(This fact is obviously true set theoretically, and is proved scheme theoretically by the computation of the canonical bundles, which gives:

$$
\left.K_{\tilde{R}}=\tilde{g}^{*} K_{R} \cdot\right)
$$

The zero set $Z_{\sigma}$ is supported away of this singular locus. For each $i$ we have a natural restriction map

$$
H^{1}\left(P_{x_{i}}, g^{*} S^{k-3} \mathcal{E} \otimes H_{x_{i}} \otimes \mathcal{I}_{Z_{\sigma}}\right) \rightarrow H^{1}\left(\tilde{R}, \tilde{g}^{*} S^{k-3} \mathcal{E} \otimes H_{x_{i}} \otimes \mathcal{I}_{Z_{\sigma}}\right),
$$

since $\tilde{R}=P_{x_{1}} \times_{G_{2}} \ldots \times_{G_{2}} P_{x_{2 k-1}}$ admits a natural morphism to $P_{x_{i}}$. Next we have by the above description of $\tilde{R}$ an isomorphism

$$
H^{1}\left(R, S^{k-3} \mathcal{E} \otimes \mathcal{E}_{\mid R} \otimes \mathcal{I}_{Z_{\sigma}}\right) \cong H^{1}\left(\tilde{R}, \tilde{g}^{*} S^{k-3} \mathcal{E} \otimes \mathcal{E} \otimes \mathcal{I}_{Z_{\sigma}}\right)
$$

and it follows that the injectivity of the map (4.40) will be a consequence of the injectivity of the map

$$
H^{1}\left(\tilde{R}, \tilde{g}^{*} S^{k-3} \mathcal{E} \otimes \mathcal{E} \otimes \mathcal{I}_{Z_{\sigma}}\right) \rightarrow \oplus_{i} H^{1}\left(\tilde{R}, \tilde{g}^{*} S^{k-3} \mathcal{E} \otimes H_{x_{i}} \otimes \mathcal{I}_{Z_{\sigma}}\right)
$$

induced by the morphisms $\tilde{g}^{*} \mathcal{E} \rightarrow H_{x_{i}}$ on $\tilde{R}$. Recall now that $Z_{\sigma} \subset R$ is defined by a section of $\mathcal{L}$ so that similarly $Z_{\sigma} \subset \tilde{R}$ is defined by a section of $\tilde{g}^{*} \mathcal{L}$. Hence we have

$$
\mathcal{I}_{Z_{\sigma}} \cong \tilde{g}^{*} \mathcal{L}^{-1}
$$

Furthermore

$$
K_{\tilde{R}}=\tilde{g}^{*} K_{R}=\tilde{g}^{*}\left(K_{G_{2 \mid R}} \otimes \mathcal{L}^{2 k-1}\right)=\tilde{g}^{*} \mathcal{L}^{k-3} .
$$

Hence the map (4.41) dualizes by Serre's duality as the map

$$
\begin{array}{r}
\oplus_{i} H^{0}\left(\tilde{R}, \tilde{g}^{*} S^{k-3} \mathcal{E}^{*} \otimes H_{x_{i}}^{*} \otimes \tilde{g}^{*} \mathcal{L} \otimes \tilde{g}^{*} \mathcal{L}^{k-3}\right) \\
\rightarrow H^{0}\left(\tilde{R}, \tilde{g}^{*}\left(S^{k-3} \mathcal{E}^{*} \otimes \mathcal{E}^{*}\right) \otimes \tilde{g}^{*} \mathcal{L} \otimes \tilde{g}^{*} \mathcal{L}^{k-3}\right)
\end{array}
$$

given by the inclusions $H_{x_{i}}^{*} \subset \tilde{g}^{*} \mathcal{E}^{*}$ on $\tilde{R}$. Since $\operatorname{det} \mathcal{E}=\mathcal{L}$, we have

$$
\mathcal{E}^{*} \otimes \mathcal{L} \cong \mathcal{E}
$$


Hence (4.42) rewrites as

$$
\oplus_{i} H^{0}\left(\tilde{R}, \tilde{g}^{*}\left(S^{k-3} \mathcal{E} \otimes \mathcal{L}\right) \otimes H_{x_{i}}^{*}\right) \rightarrow H^{0}\left(\tilde{R}, \tilde{g}^{*}\left(S^{k-3} \mathcal{E} \otimes \mathcal{E}\right)\right)
$$

given by the inclusions

$$
H_{x_{i}}^{*} \otimes \tilde{g}^{*} \mathcal{L} \subset \tilde{g}^{*} \mathcal{E}
$$

We want to show that (4.41) is injective, or that (4.44) is surjective. We already noticed that the restriction map

$$
\begin{aligned}
& H^{0}\left(G_{2}, S^{k-3} \mathcal{E} \otimes \mathcal{E}\right)=S^{k-3} H^{0}(E)^{*} \otimes H^{0}(E)^{*} \\
\rightarrow & H^{0}\left(R, S^{k-3} \mathcal{E} \otimes \mathcal{E}\right)=H^{0}\left(\tilde{R}, \tilde{g}^{*}\left(S^{k-3} \mathcal{E} \otimes \mathcal{E}\right)\right)
\end{aligned}
$$

is surjective. On the other hand, consider the 2-dimensional subspace $K_{x_{i}}^{\perp} \subset$ $H^{0}(E)^{*}$. It is obvious that it parametrizes sections of

$$
\operatorname{Ker}\left(H^{0}\left(P_{x_{i}}, g^{*} \mathcal{E}\right) \rightarrow H^{0}\left(P_{x_{i}}, H_{x_{i}}\right)\right)=H^{0}\left(P_{x_{i}}, g^{*} \mathcal{L} \otimes H_{x_{i}}^{*}\right)
$$

Hence the surjective map

$$
S^{k-3} H^{0}(E)^{*} \otimes H^{0}(E)^{*} \rightarrow H^{0}\left(\tilde{R}, \tilde{g}^{*}\left(S^{k-3} \mathcal{E} \otimes \mathcal{E}\right)\right)
$$

sends $S^{k-3} H^{0}(E)^{*} \otimes K_{x_{i}}^{\perp}$ in the subspace $H^{0}\left(\tilde{R}, \tilde{g}^{*}\left(S^{k-3} \mathcal{E} \otimes \mathcal{L}\right) \otimes H_{x_{i}}^{*}\right)$.

Now since the $x_{i}$ 's are generic, the spaces $K_{x_{i}}^{\perp}$ generate $H^{0}(E)^{*}$, hence the $S^{k-3} H^{0}(E)^{*} \otimes K_{x_{i}}^{\perp}$ 's generate $S^{k-3} H^{0}(E)^{*} \otimes H^{0}(E)^{*}$. Hence we have shown that (4.44) is surjective.

\section{Appendix}

We consider the Grassmannian $G_{2}$ of rank 2 vector subspaces of a $k+2$-dimensional vector space $V$. Let $\mathcal{L}$ be the line bundle on $G_{2}$ whose sections give the Plücker embedding. If $\mathcal{E}$ is the dual of the tautological subbundle $\mathcal{S} \subset V \otimes \mathcal{O}_{G_{2}}$, we have $\mathcal{L}=\operatorname{det} \mathcal{E}$. The cohomology groups $H^{p}\left(G_{2}, \mathcal{L}^{-q} \otimes S^{q^{\prime}} \mathcal{E}\right)$ are described in the following proposition.

Proposition 9. For $q>0, q^{\prime}>0$, we have

$$
H^{p}\left(G_{2}, \mathcal{L}^{-q} \otimes S^{q^{\prime}} \mathcal{E}\right)=0 \text { if } p \neq k, 2 k .
$$

Furthermore, for $p=k$, we have

$$
H^{p}\left(G_{2}, \mathcal{L}^{-q} \otimes S^{q^{\prime}} \mathcal{E}\right)=0 \text { if }-q+q^{\prime}+1<0,
$$

and for $p=2 k$, we have

$$
H^{p}\left(G_{2}, \mathcal{L}^{-q} \otimes S^{q^{\prime}} \mathcal{E}\right)=0 \text { if }-q+q^{\prime} \geq-k-1
$$


Proof. Let

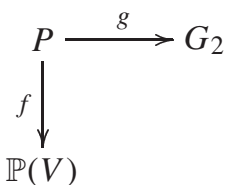

be the incidence variety. $P$ is a $\mathbb{P}^{1}$-bundle over $G_{2}$ and a $\mathbb{P}^{k}$-bundle over $\mathbb{P}(V)$. Let $H:=f^{*} \mathcal{O}_{\mathbb{P}(V)}(1)$ and let $L^{\prime}=g^{*} \mathcal{L}$. Then $\mathcal{E}=R^{0} g_{*} H$ and $S^{q^{\prime}} \mathcal{E}=R^{0} g_{*}\left(q^{\prime} H\right)$. It follows that we have

$$
H^{p}\left(G_{2}, \mathcal{L}^{-q} \otimes S^{q^{\prime}} \mathcal{E}\right)=H^{p}\left(P,-q L^{\prime}+q^{\prime} H\right)
$$

Next the line bundle $L^{\prime}$ restricts to $\mathcal{O}(1)$ on the fibers of $\pi$. It follows from this that

$$
K_{P}=-(k+1) L^{\prime}-2 H,
$$

and $K_{P / \mathbb{P}(V)}=-(k+1) L^{\prime}+k H$.

Now since $q>0$ we have $R^{l} f_{*}\left(-q L^{\prime}+q^{\prime} H\right)=0$ for $l<k$ and hence

$$
H^{p}\left(P,-q L^{\prime}+q^{\prime} H\right)=H^{p-k}\left(\mathbb{P}(V), R^{k} f_{*}\left(L^{\prime}+q^{\prime} H\right)\right) .
$$

By Serre's duality, we have

$$
\begin{gathered}
R^{k} f_{*}\left(-q L^{\prime}+q^{\prime} H\right)=\left(R^{0} f_{*}\left(q L^{\prime}-q^{\prime} H-(k+1) L^{\prime}+k H\right)\right)^{*} \\
=\left(R^{0} f_{*}\left((q-(k+1)) L^{\prime}+\left(k-q^{\prime}\right) H\right)\right)^{*} .
\end{gathered}
$$

Now we have

$$
R^{0} f_{*}\left((q-(k+1)) L^{\prime}\right)=0
$$

if $q<k+1$, and

$$
R^{0} f_{*}\left((q-(k+1)) L^{\prime}\right) \cong S^{q-k-1}\left(\Omega_{\mathbb{P}(V)}(2)\right)
$$

for $q \geq k+1$. (The isomorphism (5.47) for $q-k+1=1$ follows from the isomorphism

$$
H^{0}\left(P, L^{\prime}\right)=H^{0}\left(G_{2}, \mathcal{L}\right)=\bigwedge^{2} V^{*}=H^{0}\left(\mathbb{P}(V), \Omega_{\mathbb{P}(V)}(2)\right)
$$

and from the comparison of the kernels of the surjective evaluation maps

$$
H^{0}\left(P, L^{\prime}\right) \rightarrow H^{0}\left(f^{-1}(x), L^{\prime}\right)
$$

and

$$
\left.H^{0}\left(\mathbb{P}(V), \Omega_{\mathbb{P}(V)}(2)\right) \rightarrow \Omega_{\mathbb{P}(V)}(2)_{x} .\right)
$$


Finally we conclude from (5.45), (5.46) and (5.47) that

1. $H^{p}\left(P,-q L^{\prime}+q^{\prime} H\right)=0$ for $p<k$.

2. $H^{p}\left(P,-q L^{\prime}+q^{\prime} H\right)=0$ for $q<k+1$.

3. For $p \geq k, q \geq k+1$,

$$
H^{p}\left(P,-q L^{\prime}+q^{\prime} H\right)=H^{p-k}\left(\mathbb{P}(V), S^{q-k-1}\left(T_{\mathbb{P}(V)}(-2)\right)\left(q^{\prime}-k\right)\right) .
$$

To conclude, consider the Euler exact sequence

$$
0 \rightarrow \mathcal{O}_{\mathbb{P}(V)}(-1) \rightarrow V \otimes \mathcal{O}_{\mathbb{P}(V)} \rightarrow T_{\mathbb{P}(V)}(-1) \rightarrow 0 .
$$

It induces the exact sequences

$$
\begin{aligned}
& 0 \rightarrow S^{q-k-2} V \otimes \mathcal{O}_{\mathbb{P}(V)}\left(-q+q^{\prime}\right) \rightarrow S^{q-k-1} V \otimes \mathcal{O}_{\mathbb{P}(V)}\left(-q+q^{\prime}+1\right) \\
& \rightarrow S^{q-k-1}\left(T_{\mathbb{P}(V)}(-1)\right)\left(-q+q^{\prime}+1\right) \rightarrow 0 .
\end{aligned}
$$

Hence we conclude that the space $H^{p}\left(G_{2}, \mathcal{L}^{-q} \otimes S^{q^{\prime}} \mathcal{E}\right)$ which by the above is also isomorphic to

$$
\begin{gathered}
H^{p-k}\left(\mathbb{P}(V), S^{q-k-1}\left(T_{\mathbb{P}(V)}(-2)\right)\left(q^{\prime}-k\right)\right) \\
=H^{p-k}\left(\mathbb{P}(V), S^{q-k-1}\left(T_{\mathbb{P}(V)}(-1)\right)\left(-q+q^{\prime}+1\right)\right)
\end{gathered}
$$

is equal to 0 for $p-k \neq 0, k$ (since $p \leq 2 k$ ), and that:

- for $p-k=0$ it is 0 if $-q+q^{\prime}+1<0$;

- for $p-k=k$ it is 0 if $-q+q^{\prime} \geq-k-1$.

\section{References}

1. M. Aprodu: On the vanishing of higher syzygies of curves, preprint 2000, to appear in Math. Z.

2. E. Arbarello, M. Cornalba, Ph. Griffiths, J. Harris: Geometry of algebraic curves, Vol. 1, Grundlehren der Math. Wissenschaften 267, Springer-Verlag (1985)

3. G. Danila: Sur la cohomologie d'un fibré tautologique sur le schéma de Hilbert d'une surface, J. Alg. Geometry 10(2), 247-280 (2001)

4. S. Ehbauer: Syzygies of points in projective space and applications, in: Proceedings of the international conference, Ravello 1992, ed. by Orecchia and Chiantini, Walter de Gruyter (1994)

5. L. Ein: A remark on the syzygies of the generic canonical curve, J. Diff. Geom. 26, 361-365 (1987)

6. D. Eisenbud, J. Harris: Limit linear series, basic theory, Invent. Math. 85, 337-371 (1986)

7. M. Green. Koszul cohomology and the geometry of projective varieties, J. Diff. Geom. 19, 125-171 (1984)

8. M. Green: R. Lazarsfeld: Special divisors on curves on a $K 3$ surface, Invent. Math. 89, 357-370 (1987)

9. M. Green, R. Lazarsfeld: A simple proof of Petri's theorem on canonical curves, in: Geometry today, Giornate di Geometria Roma (1984) PM 60, Birkhäuser, pp. 129-142

10. A. Hirschowitz, S. Ramanan: New evidence for Green's conjecture on syzygies of canonical curves, Ann. Sci. Ecole Norm. sup., IV Série 31(4), 141-152 (1998)

11. R. Lazarsfeld: Brill-Noether-Petri without degenerations, J. Diff. Geom. 23, 299-307 (1986) 
12. S. Mukai: Biregular classification of Fano threefolds and Fano manifolds of coindex 3 , Proc. Nat. Acad. Sci. USA 86, 3000-3002 (1989)

13. S. Mukai: Symplectic structure on the moduli space of sheaves on an abelian or $K 3$ surface, Invent. Math. 77, 101-116 (1984)

14. F.-O. Schreyer: Syzygies of canonical curves and special linear series, Math. Ann. 275, 105-137 (1986)

15. F.-O. Schreyer: A standard basis approach to the syzygies of canonical curves, J. Reine Angew. Math. 421, 83-123 (1991)

16. M. Teixidor: Green's conjecture for the generic canonical curve, preprint 1999, unpublished

17. M. Teixidor: Green's conjecture for the generic $r$-gonal curve of genus $g \geq 3 r-7$, Duke Math. J. 111(2), 195-222 (2002)

18. C. Voisin: Sur l'application de Wahl des courbes satisfaisant la condition de BrillNoether-Petri, Acta Math. 168, 249-272 (1992)

19. C. Voisin: Courbes tétragonales et cohomologie de Koszul, J. Reine Angew. Math. 387 (1988)

20. C. Voisin: Déformation des syzygies et théorie de Brill-Noether, Proc. London Math. Soc. 3 67, 493-515 (1993) 